\title{
On A. Connes' Noncommutative Integration Theory*
}

\author{
Daniel Kastler \\ Centre de Physique Théorique, CNRS, Luminy, Case 907, F-13288 Marseille, Cedex 2, France
}

\begin{abstract}
This paper is a comment (written in a self-contained way) upon A. Connes' noncommutative integration theory. A simple observation (Lemma 9) allows the direct definition of transverse measures as functionals on the general positive random functions. Connes' first definition is then recovered by considering the transverse functions as a separating subset of the positive random functions (to which they are related by explicit natural transformations).
\end{abstract}

\section{Introduction}

The aim of this paper is to provide easier (at any rate more leisurely) access to the notion of transverse measures on groupoids developed in [1], which we believe to be connected with quantization (we hope to return to this later). Alain Connes was led to his noncommutative integration theory in his generalization of the Index Theorem to foliations on compact manifolds, by the need to integrate transversally an everywhere infinite function (the dimension of the space of harmonic forms on the generically noncompact leaves). The two major points of this theory are the following: on the one hand, it allows integration over singular quotients (e.g., non standard orbit spaces of ergodic actions), giving a precise meaning to a heuristic situation where the space shrinks whilst the function becomes infinite (tiny space). On the other, it provides the right (i.e., fully functorial) description of KMS states of convolution algebras of groupoids, these being a possibly largely universal model for noncommutative algebras, related with "geometry" via the groupoid. Consideration of the tiny space in fact enforces noncommutativity - a feature reflecting the incorporation to classical analysis of the non type I phenomena encountered in operator algebras (and in the algebraic theory of infinite quantum systems).

One of the basic ideas of Connes' integration theory is a generalization of the notion of a (real- or integer-valued) numerical function. Instead of plotting a number against the variable, one plots a standard Borel set with "cardinality"

* This work is supported in part by the National Science Foundation, Grant MCS 79-03041 
(= total measure) this number - possibly everywhere infinite - so as to build an appropriate measurable bundle. The base of this bundle is the standard space to be divided by a measurable, possibly nonregular equivalence relation (e.g. the orbit space of a nonregular ergodic action). A measurable groupoid enters this picture by means of a functor of bimeasurable bundle maps with associated maps, the groupoid elements, acting on the base as its set of units. This procedure amounts to integrating on the quotient space obtained from a well behaved "desingularization".

As in the case of groups, convolution of functions on a groupoid is obtained by convolution of the measures products of functions by "Haar measure": this is the object of Sects. 3 and 4 to follow. Section 5 aims only at motivating the concept of $\delta$-symmetric measures: it can be skipped [but for the definition (30) of the latter]. Section 6 describes the generalized notion of functions. The central Sect. 7 can either be read as it is, or alternatively, permuting Theorems 8 and 10. The functorial properties of the transverse measures are described in Sect. 8. Appendix A is technical. Appendix B describes examples and can be read after Sect.1.

\section{Measurable Groupoids}

A groupoid $\Gamma$ is a small category with inverses. Specifically: we have a set $\Gamma^{(0)}$ of objects with, to each pair $x, y \in \Gamma^{(0)}$ a (possibly void) set $\Gamma_{x}^{y}$ of morphisms $\gamma: x \rightarrow y,{ }^{1}$ $\Gamma$ being the disjoint union of all $\Gamma_{x}^{y}, x, y \in \Gamma^{(0)}$. Further, to each pair $\gamma: x \rightarrow y$, $\gamma^{\prime}: y \rightarrow z, x, y, z \in \Gamma^{(0)}$, there is a product $\gamma^{\prime} \gamma: x \rightarrow z$ with the following properties:

(i) if $\gamma: x \rightarrow y, \gamma^{\prime}: y \rightarrow z, \gamma^{\prime \prime}: z \rightarrow u, x, y, z, u \in \Gamma^{(0)}$, we have $\gamma^{\prime \prime}\left(\gamma^{\prime} \gamma\right)=\left(\gamma^{\prime \prime} \gamma^{\prime}\right) \gamma$,

(ii) to each $y \in \Gamma^{(0)}$ there is a unit $\mathbb{1}_{y}: y \rightarrow y$ with the properties $\mathbb{1}_{y} \gamma=\gamma$ and $\gamma^{\prime} \mathbb{1}_{y}=\gamma^{\prime}$ whenever $\gamma: x \rightarrow y$ and $\gamma^{\prime}: y \rightarrow z, x, y, z \in \Gamma^{(0)}$ (from this it is follows that such $\mathbb{1}_{y}$ is unique, which allows us to identify $y$ and $\mathbb{1}_{y}$ and consider $\Gamma^{(0)} \subset \Gamma$ as the set of units in $\Gamma$ ),

(iii) to each $\gamma: x \rightarrow y$ there is $\gamma^{-1}: y \rightarrow x$ with $\gamma^{-1} \gamma=x$ and $y \gamma^{-1}=y$ [from this it follows that $\gamma^{-1}$ is unique, and thus that $\left.\left(\gamma^{-1}\right)^{-1}=\gamma\right]$.

For $\gamma: x \rightarrow y$ we set $x=s(\gamma)$ (source of $\gamma$ ) and $y=r(\gamma)$ (range of $\gamma$ ). We denote $\Gamma^{(2)}=\left\{\left(\gamma, \gamma^{\prime}\right) \in \Gamma \times \Gamma ; s(\gamma)=r\left(\gamma^{\prime}\right)\right\}$ the set of multipliable pairs and, for $y \in \Gamma^{(0)}$, we set $\Gamma^{y}=r^{-1}(\{y\})$.

A measurable groupoid $\Gamma=(\Gamma, \mathbb{B})$ is a groupoid $\Gamma$ with a tribe $\mathbb{B}$ of measurable sets such that the maps $\gamma: \Gamma \rightarrow \gamma^{-1} \in \Gamma,\left(\gamma, \gamma^{\prime}\right) \in \Gamma^{(2)} \rightarrow \gamma^{\prime} \gamma \in \Gamma$ and $r, s: \Gamma \rightarrow \Gamma^{(0)}$ are all measurable $\left(\Gamma \times \Gamma\right.$ is given the product, $\Gamma^{(0)} \subset \Gamma$ and $\Gamma^{(2)} \subset \Gamma \times \Gamma$ the induced, measurable structure).

\section{Kernels}

We shall need the following notion: given two measurable spaces, $(Y, \mathbb{B}),\left(Y^{\prime}, \mathbb{B}^{\prime}\right)$ with respective sets of positive extended real valued ${ }^{2}$ measurable functions $\overline{\mathscr{F}}^{+}(Y)$,

1 We write indifferently $\gamma \in \Gamma_{x}^{y}$ or $\gamma: x \rightarrow y$

2 The positive extended real line is $\bar{R}^{+}=R^{+} \cup(+\infty)$ 
$\overline{\mathscr{F}}^{+}\left(Y^{\prime}\right)$, a kernel from $Y$ to $Y^{\prime}$ is a map $\lambda: \overline{\mathscr{F}}+(Y) \rightarrow \overline{\mathscr{F}}^{+}\left(Y^{\prime}\right)^{3}$ which is

(i) affine, i.e. $\lambda(\alpha f+\beta g)=\alpha \lambda(f)+\beta \lambda(g), \alpha, \beta \in \bar{R}^{+}, f, g \in \overline{\mathscr{F}}^{+}(Y)$,

(ii) normal, i.e. $f_{n}>f, f_{n} \in \overline{\mathscr{F}}^{+}(Y)$ implies $\lambda\left(f_{n}\right)>\lambda(f)$.

Denoting $\overline{\mathfrak{M}}^{+}(Y)$ the set of positive measures on $Y$, we note that the kernel $\lambda$ is determined by the map $y^{\prime} \rightarrow \lambda^{y^{\prime}} \in \overline{\mathfrak{M}}^{+}(Y)$, where $\lambda^{y^{\prime}}(S)=\lambda\left(\mathbb{1}_{S}\right)\left(y^{\prime}\right), S \in \mathbb{B}$ with characteristic function $\mathbb{1}_{S}$ : one has namely

$$
\lambda(f)\left(y^{\prime}\right)=\lambda^{y^{\prime}}(f)=\int d \lambda^{y^{\prime}}(y) f(y), y^{\prime} \in Y^{\prime}, f \in \overline{\mathscr{F}}^{+}(Y) .
$$

In fact, the kernels $\lambda: Y \rightarrow Y^{\prime}$ could be defined as maps $\left(y^{\prime}, S\right) \in Y^{\prime} \times \mathbb{B} \rightarrow \lambda^{y^{\prime}}(S)$ such that $\lambda^{y^{\prime}}(\cdot) \in \overline{\mathfrak{M}}^{+}(Y), y^{\prime} \in Y^{\prime}$, and $\lambda \cdot(S) \in \overline{\mathscr{F}}^{+}\left(Y^{\prime}\right), S \in \mathbb{B}$, (unbounded generalizations of transition probabilities). Given kernels $\lambda: Y \rightarrow Y^{\prime}$ and $\mu: Y^{\prime} \rightarrow Y^{\prime \prime}$, it is obvious that $\mu \lambda=\mu \circ \lambda$ is a kernel from $Y$ to $Y^{\prime \prime}$.

Given measurable spaces $Y, Y^{\prime}, X$ with $Y, Y^{\prime}$ respectively fibered by $\pi: Y \rightarrow X$ and $\pi^{\prime}: Y^{\prime} \rightarrow X$ (i.e. $\pi$ and $\pi^{\prime}$ are measurable onto), a kernel $\lambda: Y \rightarrow Y^{\prime}$ is called fibered by $\pi$ and $\pi^{\prime}$ whenever the restriction of $\lambda(f), f \in \overline{\mathscr{F}}^{+}(Y)$, to the fibre $\pi^{\prime-1}(x)$ of $x$, $x \in X$, depends only on the restriction of $f$ to the corresponding fibre $\pi^{-1}(x)$. It is easily seen that this is the case if and only if ${ }^{4}$

$$
\lambda((\varphi \circ \pi) f)=\left(\varphi \circ \pi^{\prime}\right) \lambda(f), \quad f \in \overline{\mathscr{F}}^{+}(Y), \varphi \in \overline{\mathscr{F}}^{+}(X) .
$$

It follows that the product of fibered kernels are fibered kernels. A special case of fibered kernels is obtained by considering measurable spaces $Y, X$, with $Y$ fibered by $\pi: Y \rightarrow X$, and $X$ trivially fibered by its identity map $\operatorname{id}_{X}$ : a kernel $\lambda: Y \rightarrow X$ is then fibered by $\pi$ and id $_{X}$ iff the measure $\lambda^{x} \in \overline{\mathfrak{M}}^{+}(Y)$ is carried by the fibre $\pi^{-1}(x)$ for each $x \in X$.

A kernel $\lambda: Y \rightarrow Y^{\prime}$ is called $\sigma$-finite (respectively proper) whenever there is a sequence $\left\{B_{n}\right\}$ of measurable subsets of $Y$ such that $\bigcup_{n} B_{n}=Y$ and $\lambda\left(\mathbb{1}_{B_{n}}\right)$ is finite (respectively bounded). One easily sees that the existence of a nowhere vanishing $f \in \overline{\mathscr{F}}^{+}(Y)$ with $\lambda(f)$ finite (respectively bounded) entails that $\lambda$ is $\sigma$-finite (respectively means that $\lambda$ is proper): consider indeed $B_{n}=\left\{y \in Y ; f(y)>2^{-n}\right\}$ and $f=\sum_{n=1}^{\infty} 2^{-n}\left\|\lambda\left(B_{n}\right)\right\|_{\infty} \mathbb{1}_{B_{n}}$. Note also that $f$ can always be chosen bounded, and the $B_{n}$ nondecreasing.

\section{Convolution on Measurable Groupoids}

A groupoid $\Gamma$ is analogous to a group in that it possesses an associative product for which each element is invertible, but the product is not everywhere defined: for $\gamma$, $\gamma^{\prime} \in \Gamma, \gamma \gamma^{\prime}$ exists iff $s(\gamma)=r\left(\gamma^{\prime}\right)$. This suggests the following formal extension to groupoids of the notion of convolution of functions on a discrete group:

$$
(f * g)(\gamma)=\sum_{\gamma^{\prime} \in \Gamma, r\left(\gamma^{\prime}\right)=r(\gamma)} f\left(\gamma^{\prime}\right) g\left(\gamma^{\prime-1} \gamma\right) .
$$

3 We shall use the shorthand: $\lambda: Y \rightarrow Y^{\prime}$

4 In the case where the points of $X$ are measurable. Otherwise condition (2) is stronger and should be taken as defining kernels: $X \rightarrow Y$ fibered by $\pi$ and $\pi^{\prime}$ 
For discrete groupoids (isomorphic to $\mathbb{N}$ as measurable spaces) (3a) is a suitable definition of convolution. In the general case however, it has to be replaced by

$$
\left(f_{\lambda}^{*} g\right)(\gamma)=\int d \lambda^{r(\gamma)}\left(\gamma^{\prime}\right) f\left(\gamma^{\prime}\right) g\left(\gamma^{\prime-1} \gamma\right), \quad f, g \in \overline{\mathscr{F}}^{+}(\Gamma),
$$

where $\lambda^{r(\gamma)}$ (or, for that matter, $f \lambda^{r(\gamma)}$ ) is a positive measure on $\Gamma$ carried by $\Gamma^{r(\gamma)}=r^{-1}(r\{\gamma\})$. We see that convolution as defined by $(3 b)$ is relative to the specification of what we shall call a $\Gamma$-kernel $\lambda$, i.e. a kernel $\lambda: \Gamma \rightarrow \Gamma^{(0)}$ fibered by $r$ and $\mathrm{id}_{\Gamma^{(0)}}$ (cf. end of preceding section) $)^{5}$. Definition (3b) then amounts to

$$
f_{\lambda}^{*} g=f \lambda * g, f, g \in \overline{\mathscr{F}}^{+}(\Gamma),
$$

where we define as follows the convolution of a $\Gamma$-kernel $\lambda$ and a function $f \in \overline{\mathscr{F}}^{+}(\Gamma)$ :

$$
(\lambda * f)(\gamma)=\int d \lambda^{r(\gamma)}\left(\gamma^{\prime}\right) f\left(\gamma^{\prime-1} \gamma\right), \quad \gamma \in \Gamma,
$$

and where $f \lambda$ is the kernel: $\Gamma \rightarrow \Gamma^{0}$ defined by:

$$
\left.\begin{array}{l}
(f \lambda)^{y}=f \lambda^{y}, y \in \Gamma^{(0)} \\
\text { (equivalently } \left.(f \lambda)(g)=\lambda(f g), g \in \overline{\mathscr{F}}^{+}(\Gamma)\right) .
\end{array}\right\}
$$

In order however that the function $\lambda * f$ in (4) be measurable, we need to assume $\sigma$-finiteness of $\lambda$ (or equivalently properness see Lemma 2 below). We then have

Proposition 1. Let, for $\lambda$ a $\Gamma$-kernel and for $f \in \overline{\mathscr{F}}^{+}(\Gamma)$

$$
\begin{aligned}
& \left(L_{\lambda} f\right)(\gamma)=\int d \lambda^{r(\gamma)}\left(\gamma^{\prime}\right) f\left(\gamma^{\prime-1} \gamma\right)=(\lambda * f)(\gamma), \\
& \left(R_{\lambda} f\right)(\gamma)=\int d \lambda^{s(\gamma)}\left(\gamma^{\prime}\right) f\left(\gamma \gamma^{\prime}\right) .
\end{aligned}
$$

If $\lambda$ is $\sigma$-finite these formulae define kernels $L_{\lambda}, R_{\lambda}$ from $\Gamma$ to $\Gamma$ related by

where

$$
L_{\lambda} f=\left(R_{\lambda} \tilde{f}\right)^{\sim}, \quad f \in \overline{\mathscr{F}}^{+}(\Gamma),
$$

$$
\tilde{f}(\gamma)=f\left(\gamma^{-1}\right), \quad \gamma \in \Gamma .
$$

Moreover one has the following properties

$$
\begin{gathered}
\lambda(\tilde{f})=\left.(\lambda * f)\right|_{\Gamma^{(0)},}, f \in \overline{\mathscr{F}}^{+}(\Gamma), \\
L_{\lambda} L_{\mu}=L_{\lambda R_{\mu}}, \\
\left.R_{\lambda}((\varphi \circ r) f)=(\varphi \circ r) R_{\lambda} f, \quad f \in \overline{\mathscr{F}}^{+}(\Gamma), \varphi \in \overline{\mathscr{F}}^{+}\left(\Gamma^{(0)}\right),\right\} \\
L_{\lambda}((\varphi \circ s) f)=(\varphi \circ s) L_{\lambda} f, \\
\lambda(\mu * f)=\mu(\lambda * \tilde{f}), \quad f \in \overline{\mathscr{F}}^{+}(\Gamma),
\end{gathered}
$$

where $\lambda$ and $\mu$ are any $\sigma$-finite $\Gamma$-kernels.

Property (9) suggests the notation ${ }^{6}$

$5 \quad \Gamma$-kernels are thus kernels $\lambda: \Gamma \rightarrow \Gamma^{(0)}$ such that $\lambda((\varphi \circ r) f)=\varphi \lambda(f), f \in \overline{\mathscr{F}}^{+}(\Gamma), \varphi \in \overline{\mathscr{F}}^{+}\left(\Gamma^{(0)}\right)$

6 Note that $\lambda R_{\mu}$ is a $\Gamma$-kernel since $R_{\mu}$ is fibered by $r$ and $r$, and $\lambda$ by $r$ and id $\Gamma^{(0)}$. The third equation in (12) generalizes to $\left(\lambda_{1} * \lambda_{2} * \ldots * \lambda_{n}\right)^{y}(f)=\int d \lambda_{1}^{y}\left(\gamma_{1}\right) d \lambda_{2}^{s\left(\gamma_{1}\right)}\left(\gamma_{2}\right) d \lambda_{n}^{s\left(\gamma_{n-1}\right)}\left(\gamma_{n}\right) \tilde{f}\left(\gamma_{1} \gamma_{2} \ldots \gamma_{n}\right), \lambda_{1}, \ldots, \lambda_{n}$ $\sigma$-finite $\Gamma$-kernels, $f \in \overline{\mathscr{F}}^{+}(\Gamma), y \in \Gamma^{(0)}$ 


$$
\lambda R_{\mu}=\lambda * \mu, \quad \text { so } \quad\left\{\begin{array}{l}
\lambda *(\mu * f)=(\lambda * \mu) * f, f \in \overline{\mathscr{F}}^{+}(\Gamma), \\
(\lambda * \mu)(f)=\left(\lambda R_{\mu}\right)(f) .
\end{array}\right.
$$

The proof that $L_{\lambda} f$ (or, for that matter $R_{\lambda} f$ ) is measurable as a consequence of the fact that $\lambda$ is $\sigma$-finite is somewhat technical and deferred to Appendix A. Properties (6a) through (11) follow from easy computations. If $\gamma=x \in \Gamma^{(0)}$ in (4) one has $r(\gamma)=x$ and $\gamma^{\prime-1} \gamma=\gamma^{\prime-1}$, whence (8). Equation (9) is checked as follows:

$$
\begin{aligned}
\left(L_{\lambda} L_{\mu} f\right)(\gamma) & =\int d \lambda^{r(\gamma)}\left(\gamma^{\prime}\right) \int d \mu^{r\left(\gamma^{\prime-1} \gamma\right)}\left(\gamma^{\prime \prime}\right) f\left(\gamma^{\prime \prime}-1 \gamma^{\prime-1} \gamma\right) \\
& =\int d \lambda^{r(\gamma)}\left(\gamma^{\prime}\right) \int d \mu^{s\left(\gamma^{\prime}\right)}\left(\gamma^{\prime \prime}\right) f\left(\left(\gamma^{\prime} \gamma^{\prime \prime}\right)^{-1} \gamma\right) \\
& =\left\langle\left(\lambda R_{\mu}\right)^{r(\gamma)}, \tilde{f(}\left(\gamma^{-1} \cdot\right)\right\rangle=\left(L_{\lambda R_{\mu}} f\right)(\gamma) .
\end{aligned}
$$

Equation (10) is immediate from the definitions (4a) and (6). As for (11) we have

$$
\begin{aligned}
\lambda^{y}(\mu * f) & =\int d \lambda^{y}(\gamma) \int d \mu^{r(\gamma)}\left(\gamma^{\prime}\right) f\left(\gamma^{\prime-1} \gamma\right) \\
& =\int d \lambda^{y}(\gamma) \int d \mu^{y}\left(\gamma^{\prime}\right) f\left(\gamma^{\prime-1} \gamma\right),
\end{aligned}
$$

whence the result, since interchange of integrations is licit by the $\sigma$-finiteness assumption for $\lambda$ and $\mu^{7}$.

Lemma 2. Let $\Gamma$ be a measurable groupoid and let $\lambda$ be a $\Gamma$-kernel. The following are equivalent:

(i) $\lambda$ is $\sigma$-finite,

(ii) there is a nowhere vanishing $f \in \overline{\mathscr{F}}^{+}(\Gamma)$ with $\lambda f$ finite,

(iii) there is a nowhere vanishing $f \in \overline{\mathscr{F}}^{+}(\Gamma)$ with $\lambda f$ bounded,

(iv) $\lambda$ is proper, i.e. there is a sequence $\left\{B_{n}\right\}$ of measurable subsets of $\Gamma$ such that $\bigcup_{n} B_{n}=\Gamma$ and $\lambda\left(B_{n}\right)$ is bounded for all $n$.

Further, if $\lambda$ is $\sigma$-finite, the functions $f$ in (ii) and (iii) can be chosen bounded; and there is a $g \in \overline{\mathscr{F}}^{+}(\Gamma)$ with $^{8}$

$$
\lambda(g)=\mathbb{1}_{S_{\lambda}}, S_{\lambda}=\left\{x \in \Gamma^{(0)} ; \lambda^{x} \neq 0\right\} .
$$

Proof. We noted at the end of Sect. 2 the implications (iv) $\Leftrightarrow$ (iii) $\Rightarrow$ (ii) $\Rightarrow$ (i) for general kernels $\lambda$. We thus need only to check that (i) $\Rightarrow$ (iii).

(i) $\Rightarrow$ (iii): for $n, m \in \mathbb{N}$ consider $A_{m n}=\left\{y \in \Gamma^{(0)} ; \lambda^{y}\left(B_{n}\right)<m\right\}$. For a fixed $n$ the $A_{m n}$ are increasing towards $\Gamma^{(0)}$, thus the $r^{-1}\left(A_{m n}\right)$ are increasing towards $\Gamma$. Thus the $B_{m n}^{\prime}=B_{n} \cap r^{-1}\left(A_{m n}\right)$ are measurable sets of $\Gamma$ covering $\Gamma$ and such that $\lambda\left(B_{m n}^{\prime}\right)<m$. The function $f=\sum_{n m} m^{-1} 2^{-(n+m)} \mathbb{1}_{B_{n m}^{\prime}}$ then fulfills (iii).

We now show the existence of $g$ fulfilling (15). Let $f$ be as in (ii) and take $g=(\varphi \circ r) f$ with $\varphi(g)=\lambda^{y}(f)^{-1}$ for $y \in S_{\lambda}$ and $\varphi=1$ for $y \notin S_{\lambda}$ (observe that for $y \in S_{\lambda}, \lambda^{y}(f)>0$ since $\lambda^{y} \neq 0$ and $f$ is nowhere vanishing). Our conclusion follows from

$$
\lambda(g)=\lambda((\varphi \circ r) f)=(\varphi \circ r) \lambda(f) .
$$

7 Note that Eqs. (8) and (10) hold without $\sigma$-finiteness assumption

8 We denoted by $\mathbb{1}_{S}$ the characteristic function of the set $S$ and call a function finite whenever all its values are finite

Note that (since the proof of Lemma 2 makes no use of the fact that $\Gamma$ is a groupoid with set of units $\left.\Gamma^{(0)}\right)$ the result in fact holds for any kernel $\lambda: Y \rightarrow X$ fibered by $r$ and $i d_{X}$, with $r$ a measurable surjection $Y \rightarrow X$ 


\section{Transverse Functions}

Our next question is whether groupoids give rise to *-algebras in the same manner as groups ${ }^{9}$. Specifically, given a $\sigma$-finite $\Gamma$-kernel $\lambda$, we want to know when the convolution ${ }_{\lambda}^{*}$ which it defines is associative and symmetric (in the sense that $\left.\left(f_{\lambda}^{*} g\right)^{\sim}=\tilde{g} \underset{\lambda}{*} \widetilde{f}\right)$ so as to yield a $*$-algebra with the product $\underset{\lambda}{*}$ and the adjoint operation $f^{*}=\tilde{f}$. We do not expect that this is the case for a general $\Gamma$-kernel $\lambda$, since in the group case convolution is generally neither associative nor symmetric (unless the measure at hand is the Haar measure).

Proposition 3. Let $\Gamma$ be a measurable groupoid and let $v$ be a $\Gamma$-kernel. The following are equivalent

(i) $v^{r(\gamma)}=\gamma v^{s(\gamma)}, \gamma \in \Gamma$ (left invariance, $\gamma$ denotes multiplication to the left by $\left.\gamma\right)$,

(ii) $v * f=v(\tilde{f}) \circ s, f \in \overline{\mathscr{F}}^{+}(\Gamma)$,

(iii) $(f * g)^{\sim}=\tilde{g} * \tilde{f}, f, g \in \overline{\mathscr{F}}+(\Gamma)(\sim$-symmetry),

(iv) $\lambda * f v=(\lambda * f) v, f \in \overline{\mathscr{F}}^{+}(\Gamma), \lambda$ any $\sigma$-finite $\Gamma$-kernel.

Further, in this case, and if $v$ is $\sigma$-finite, the convolution $*$ is associative.

Statement (i) makes sense as follows: left multiplication by $\gamma$ maps $\Gamma^{s(\gamma)}$, which carries $v^{s(\gamma)}$, onto $\Gamma^{r(\gamma)}$, which carries $v^{r(\gamma)}$.

Note that the $\sim$-symmetry (iii) is equivalent to the left invariance (i) and suffices to insure associativity.

The $\sigma$-finite $\Gamma$-kernels $v$ fulfilling (i) through (iv) above are called the transverse functions (or Haar systems) on $\Gamma$. Their set is denoted $\mathscr{E}^{+}$.

Proof. Condition (i) means that

$$
\int d v^{r(\gamma)}\left(\gamma^{\prime}\right) f\left(\gamma^{\prime}\right)=\int d v^{s(\gamma)}\left(\gamma^{\prime}\right) f\left(\gamma \gamma^{\prime}\right), f \in \overline{\mathscr{F}}^{+}(\Gamma), \gamma \in \Gamma,
$$

and can be written formally

$$
d v^{r(\gamma)}\left(\gamma^{\prime}\right)=d v^{s(\gamma)}\left(\gamma^{-1} \gamma^{\prime}\right), \gamma, \gamma^{\prime} \in \Gamma
$$

Condition (ii) then amounts to writing (17) for the function $\tilde{f}\left(\gamma^{-1} \cdot\right)$. Owing to the definition ( $3 \mathrm{~b})$, condition (iii) reads

$$
\int d v^{s(\gamma)}\left(\gamma^{\prime}\right) f\left(\gamma^{\prime}\right) g\left(\gamma^{\prime-1} \gamma^{-1}\right)=\int d \nu^{r(\gamma)}\left(\gamma^{\prime}\right) g\left(\gamma^{\prime-1}\right) f\left(\gamma^{-1} \gamma^{\prime}\right)
$$

which amounts to writing (17) for the function $f\left(\gamma^{-1} \cdot\right) \tilde{g}$ : thus (iii) is obviously equivalent to (i). To prove the equivalence (iv) $\Leftrightarrow$ (iii), we notice that the latter is equivalent to

$$
\lambda\left((f \underset{v}{*} \tilde{g})^{\sim}\right)=\lambda(g \underset{v}{*} \tilde{f}), f, g \in \overline{\mathscr{F}^{+}}(\Gamma), \lambda \text { any } \Gamma \text {-kernel. }
$$

Now using (8) twice, the left hand side reads

$$
\left.\lambda * f v * \tilde{g}\right|_{\Gamma^{(0)}}=(\lambda * f v)(g),
$$

whilst the right handside equals [using (11) and (6)]:

$$
\lambda(g v * \tilde{f})=g v(\lambda * f)=[(\lambda * f) v](g) .
$$

9 And, more trivially, as spaces. For these special examples of groupoids, see Appendix 2 below 
Now (iv) entails immediately the associativity of $*$ :

$$
(f * g) \underset{v}{*} h=(f v * g) v * h=f v * g v * h=f_{v} *(g * h) .
$$

Proposition 4. Let $v_{0} \in \mathscr{E}^{+}$be faithful in the sense that $v_{0}^{y} \neq 0$ for all $y \in \Gamma^{(0)}$ (in other terms $\left.S_{v}=\Gamma^{(0)}\right)$. Then each $v \in \mathscr{E}^{+}$is of the form $v_{0} * \lambda, \lambda$ a $\Gamma$-kernel. Specifically

$$
v=v_{0} * \tilde{g} v, g \in \overline{\mathscr{F}}^{+}(\Gamma), v_{0}(g)=\mathbb{1}_{\Gamma^{(0)}} .
$$

Proof. Since $S_{v}=\Gamma^{(0)}$, there is a $g \in \overline{\mathscr{F}}^{+}(\Gamma)$ with $v_{0}(g)=\mathbb{1}_{\Gamma^{(0)}}$ (cf. Lemma 2). Thus $v * \tilde{g}=\mathbb{1}_{\Gamma^{(0)} \circ S}=\mathbb{1}_{\Gamma}$. Thus $v_{0} * \tilde{g} v=\left(v_{0} * \tilde{g}\right) v=v$ (we used (ii) and (iv) in Proposition 3).

\section{KMS Weights and $\delta$-Symmetric Measures on $\Gamma^{10}$}

Now that we have a $*$-algebraic structure, we would like to define interesting states or weights, e.g. traces or KMS weights. As we know, the latter can be defined by left Hilbert algebras. We are thus led to look for scalar products on (appropriate subsets) of $\overline{\mathscr{F}}+(\Gamma)$. In order to define a positive definite scalar product, it is natural to try an expression of the form

$$
(f, g)=m(\overline{f g})
$$

where $m$ is a measure on $\Gamma$. Furthermore, it is natural to choose $m$ as obtained from a "desintegration"

$$
m=M \circ v
$$

with respect to a faithful $v \in \mathscr{E}^{+}, M$ a measure on $\Gamma^{(0)}$. Now the scalar product (23) with $m$ given by (24) has already the "right Hilbert algebra property"

$$
(f \underset{v}{*} h, g)=\left(f, g * \underset{v}{*} h^{*}\right),
$$

we have namely, using (5) and (8):

$$
(f, g)=M(v(\overline{f g}))=M(g v(\bar{f}))=M\left\{\left.\left(g \underset{v}{*} f^{*}\right)\right|_{\Gamma^{(0)}}\right\} .
$$

The same calculation performed "on the left" yields

$$
(f, g)=M((\overline{f v})(g))=M\left\{\left.(\bar{f} * \tilde{g})\right|_{\Gamma^{(0)}}\right\} .
$$

Therefore we see that we have the "left Hilbert algebra property"

$$
(h * f, g)=\left(f, h_{v}^{*} \underset{v}{*} g\right),
$$

(thus a Hilbert algebra yielding a trace) if the measure $m=M \circ v$ is "symmetric" in the sense

$$
M \circ v(f)=M \circ v(\tilde{f}), \quad f \in \overline{\mathscr{F}}^{+}(\Gamma) .
$$

This is what is needed to obtain a trace. We can generalize that in order to define KMS weights. For this we first define a modular function on $\Gamma$ to be a

10 This section is not a prerequisite for the sections to follow on noncommutative integration. We inserted it for motivating the notion of $\delta$-symmetric measures on $\Gamma$. The hurried reader can skip it, retaining only the definition of modular functions. For the notion of KMS weights, see [2] 
function $\delta: \Gamma \rightarrow R_{*}^{+}$such that $\delta\left(\gamma^{\prime-1} \gamma\right)=\delta\left(\gamma^{\prime}\right)^{-1} \delta(\gamma)$, whenever $\gamma, \gamma^{\prime} \in \Gamma$ are such that $r\left(\gamma^{\prime}\right)=r(\gamma)^{11}$. Given such a $\delta$ we now consider measures $M \circ v$ which are $\delta$-symmetric in the following sense

$$
M \circ v(f)=M \circ v\left(\delta^{-1} \tilde{f}\right), \quad f \in \overline{\mathscr{F}}^{+}(\Gamma) .
$$

We now have

Proposition 5. Let $\Gamma$ be a measurable groupoid, with $v$ a faithful transverse function on $\Gamma$. Let $M$ be a positive measure on $\Gamma^{(0)}$ fulfilling (30), and let $m=M \circ v$.

Define, on the set $\mathscr{F}(\Gamma)$ of finite measurable functions on $\Gamma$,

$$
\left.\begin{array}{rl}
f^{\#} & =\delta^{-1} f^{*}=S f, \\
\text { i.e. } f^{\#}(\gamma) & =\delta^{-1}(\gamma) \bar{f}\left(\gamma^{-1}\right), \gamma \in \Gamma, f \in \mathscr{F}(\Gamma),
\end{array}\right\}
$$

and let

$$
\mathscr{C}=\left\{f \in \overline{\mathscr{F}}(\Gamma) ; f, f^{\#} \in L^{2}(\Gamma, m) ;\|f\|<\infty\right\},
$$

where

$$
\|f\|=\operatorname{Sup}\{v(|J f|), v(|J f| \tilde{)})\}, f \in \mathscr{F}(\Gamma)
$$

with

$$
J f=\delta^{-1 / 2} f^{*}=\delta^{-1 / 2} \tilde{\tilde{f}}
$$

Then $\mathscr{C}$ with the multiplication $\underset{v}{*}$, the left involution $\#$, and the scalar product (23), is a left Hilbert algebra with corresponding modular involution J, right involution * and modular operator $\Delta$ given by

$$
\Delta f=\delta f, \quad f \in \mathscr{C} .
$$

Since this theorem is quoted only for motivating the introduction of $\delta$-symmetric measures, we merely sketch the proof. First note that the operations,$- \sim$ and $*$ on measurable functions mutually commute; and that one has $S=J \Delta^{1 / 2}=\Delta^{-1 / 2} J$, $F=J \Delta^{-1 / 2}=\Delta^{1 / 2} J, S^{2}=F^{2}=J^{2}=\mathbb{1}$ (we set $\left.\Delta^{s} f=\delta^{s} f, f \in \mathscr{F}(\Gamma), s \in \mathbb{R}\right)$. Let $\mathscr{A} \#$ be the set of $f \in \mathscr{F}^{+}(\Gamma)$ such that $\|f\|<\infty$ and let $\mathscr{A}=J \mathscr{A}^{\#}$ : these are topological *-algebras under the operations $(\underset{v}{*}, \#)$ respectively $(\underset{v}{*}, *)$; and $J$ is an antilinear bijection $\mathscr{A} \# \leftrightarrow \mathscr{A}$ reversing the product $*$ and exchanging $\#$ and $*$, moreover antiunitary for the scalar product $(23)^{12}$. For checking those facts one notices that for $f, g \in \mathscr{F}(\Gamma), v \in \mathscr{E}^{+}$, and $\lambda$ a $\Gamma$-kernel, $\|(g \lambda)(f)\|_{\infty} \leqq\|g\|_{\infty} \cdot\|\lambda(f)\|_{\infty}$ and $v(f * g)=[(v * \tilde{g}) v](f)$. The third fact we need is the boundedness of $\pi(f): \pi(f) \xi$ $=f_{v}^{*} \xi, \xi \in L^{2}(\Gamma, m), f \in \mathscr{A}$, as a Hilbert space operator. Via $J$ this follows from the boundedness of $\tilde{\pi}(f): \tilde{\pi}(f) \xi=\xi * f, f \in \mathscr{A}$. Now since

$$
\left.\begin{array}{rl}
L^{2}(\Gamma, m) & =\int_{\oplus} L^{2}\left(\Gamma^{y}, v^{y}\right) d M(y), \\
\tilde{\pi}(f) & =\int_{\oplus} \tilde{\pi}^{y}(f) d M(y),
\end{array}\right\}
$$

$11 R_{*}^{+}$denote the set of strictly positive real numbers. Modular functions on groupoids are the analogue of a positive characters of groups. As such they enjoy the properties $\delta(\lambda * f)=\delta \lambda * \delta f$, $\delta(\lambda * \mu)=\delta \lambda * \delta \mu, \lambda, \mu \Gamma$-kernels, $f \in \overline{\mathscr{F}}^{+}(\Gamma)$

12 The preclosedness of $S$ on $\mathscr{A}$ easily follows from the fact that $S$ is the restriction of $J \Delta^{1 / 2}$ with $\Delta$ selfadjoint positive and $J$ antiunitary 
with

$$
\left\{\tilde{\pi}^{y}(f) \xi\right\}(\gamma)=\int d v^{y}\left(\gamma^{\prime}\right) \xi\left(\gamma^{\prime}\right) f\left(\gamma \gamma^{\prime}\right),
$$

it follows from known facts about norms of Hilbert space operators defined by kernels that $\|\tilde{\pi}(f)\| \leqq\|J f\|_{v}$.

\section{Positive Random Variables ( $\Gamma-\delta$-Functions)}

We now describe the generalization of the notion of positive measurable function announced in the Introduction.

Let $\Gamma$ be a measurable groupoid with $\Gamma^{(0)}$ the corresponding set of units and $\delta$ a modular function on $\Gamma$. We denote by $\mathscr{L}$ the category of measurable spaces isomorphic either to $[0,1]$, or to $\mathbb{N}$, with the bimeasurable ${ }^{13}$ maps as morphisms (these two cases correspond respectively to real-, respectively integer-valued "functions").

A measurable $\Gamma$-functor $\mathbb{F}=(Z, \pi, F)$ is the specification of

(i) a functor $F: \Gamma \rightarrow \mathscr{L}$, i.e. assignments $x \in \Gamma^{(0)} \rightarrow F_{x}, F_{x}$ a measurable space as above; $\gamma \in F \rightarrow F(\gamma), F(\gamma)$ a bimeasurable map: $F_{s(\gamma)} \rightarrow \Gamma_{r(\gamma)}$, with the properties $F(x)=\mathrm{id}_{F_{x}}$, and $F\left(\gamma^{\prime-1} \gamma\right)=F\left(\gamma^{\prime}\right)^{-1} F(\gamma)$ whenever $r\left(\gamma^{\prime}\right)=r(\gamma)$,

(ii) a standard measurable space $\left(Z, \mathbb{B B}_{Z}\right)$ (the total space of $\mathbb{F}$ ) with a measurable surjection $\pi: Z \rightarrow \Gamma^{(0)}$ such that

(a) for each $x \in \Gamma^{(0)}, \pi^{-1}(x)$ is a measurable subspace of $Z$ isomorphic to $F_{x}$,

(b) the $\operatorname{map}(\gamma, z) \rightarrow F(\gamma) z$ is measurable from $\{(\gamma, z) \in \Gamma \times Z ; s(\gamma)=\pi(z)\}$ to $Z$.

We henceforth write for shortness $F(\gamma) z=\gamma z, z \in Z, \gamma \in \Gamma$.

A positive random variable on $\Gamma$ with modular function $\delta$ (or, for shortness a $\Gamma$ - $\delta$-function $) \alpha=(\mathbb{F}, \alpha)=(Z, \pi, F, \alpha)$ is the specification of

(i) a measurable $\Gamma$-functor $\mathbb{F}=(Z, \pi, F)$ in the above sense,

(ii) a $\sigma$-finite kernel $\alpha: Z \rightarrow \Gamma^{(0)}$ fibered by $\pi$ and $\operatorname{id}_{\Gamma^{(0)}}$ and $\delta$-equivariant, i.e. one has

$$
\alpha((\varphi \circ \pi) u)=\varphi \alpha(u), u \in \overline{\mathscr{F}}^{+}(Z), \varphi \in \overline{\mathscr{F}}^{+}\left(\Gamma^{(0)}\right)
$$

and

$$
\alpha^{r(\gamma)}=\delta^{-1}(\gamma) \gamma \alpha^{s(\gamma)}, \gamma \in \Gamma
$$

We will in fact concentrate attention to the proper $\Gamma$ - $\delta$-functions $\alpha=(\mathbb{F}, \alpha)$, that is those whose functor $\mathbb{F}=(Z, \pi, F)$ is proper in the sense that to each faithful $v \in \mathscr{E}^{+}$, there should be a $u \in \overline{\mathscr{F}}^{+}(Z)$ with $v * u=\mathbb{1}_{Z}$ where we define ${ }^{14}$

$$
(\lambda * u)(z)=\int d \lambda^{\pi(z)}(\gamma) u\left(\gamma^{-1} z\right),\left\{\begin{array}{l}
\lambda a \Gamma \text {-kernel } \\
u \in \mathscr{\mathscr { F }}^{+}(Z) \\
z \in Z
\end{array}\right.
$$

13 I.e. measurable invertible with measurable inverse

14 We will alternatively write $\lambda * u=F_{\lambda} u$ in analogy with (4a), hereby obtaining a kernel $F_{\lambda}: Z \rightarrow Z$ in the case of a $\sigma$-finite $\lambda$ : for this and alternative characterizations of proper functions, see Proposition 7 below 
Given two $\Gamma$-functors $\mathbb{F}=(Z, \pi, F), \mathbb{F}^{\prime}=\left(Z^{\prime}, \pi^{\prime}, F^{\prime}\right)$ a natural transformation $\varrho$ from $\mathbb{F}$ to $\mathbb{F}^{\prime}$ is a kernel $\varrho: Z^{\prime} \rightarrow Z$ which is

(i) fibered by $\pi^{\prime}$ and $\pi$ :

$$
\varrho\left(\left(\varphi \circ \pi^{\prime}\right) u^{\prime}\right)=(\varphi \circ \pi) \varrho\left(u^{\prime}\right), u^{\prime} \in \overline{\mathscr{F}}^{+}\left(Z^{\prime}\right), \varphi \in \overline{\mathscr{F}}^{+}\left(\Gamma^{(0)}\right),
$$

(ii) equivariant:

$$
\varrho^{\gamma z}=\gamma \varrho^{z}, \gamma \in \Gamma, z \in Z, r(\gamma)=\pi(z)
$$

(iii) unital: ${ }^{15}$

$$
\varrho\left(\mathbb{1}_{Z^{\prime}}\right)=\mathbb{1}_{Z}
$$

Accordingly we have the property

$$
\left.\begin{array}{rl}
\lambda * \varrho\left(u^{\prime}\right) & =\varrho\left(\lambda * u^{\prime}\right), \\
\text { i.e. } F_{\lambda} \circ \varrho & =\varrho \circ F_{\lambda}
\end{array}\right\} u^{\prime} \in \overline{\mathscr{F}}^{+}\left(Z^{\prime}\right), \lambda \text { a } \Gamma \text {-kernel, }
$$

indeed, from (i) and (ii)

$$
\begin{aligned}
\left\{\lambda * \varrho\left(u^{\prime}\right)\right\}(z) & =\int d \lambda^{\pi(z)}(\gamma) \int d \varrho^{\gamma^{-1} z}\left(z^{\prime}\right) u^{\prime}\left(z^{\prime}\right) \\
& =\int d \lambda^{\pi(z)}(\gamma) \int d \varrho^{z}\left(z^{\prime}\right) u^{\prime}\left(\gamma^{-1} z^{\prime}\right) \\
& =\int d \varrho^{z}\left(z^{\prime}\right) \int d \lambda^{\pi(z \prime)}(\gamma) u^{\prime}\left(\gamma^{-1} z^{\prime}\right) .
\end{aligned}
$$

Amongst other things (44) implies, together with (i), that if there is a natural transformation $\varrho$ from $\mathbb{F}$ to $\mathbb{F}^{\prime}$ and $\mathbb{F}^{\prime}$ is proper, so is $\mathbb{F}$.

The generalization of the notion of function afforded by the above definitions is two-fold:

1) As announced in the Introduction, the entity plotted against the variable is not a number but a "set with cardinality that number" where the "cardinality" of the set $F_{x}, x \in \Gamma^{(0)}$ is $\alpha^{x}\left(F_{x}\right)$ (possibly $+\infty$ ).

2) The variable ranges in fact in the quotient $\Gamma^{(0)} / \sim$ of the "base" $\Gamma^{(0)}$ of $Z$ by the equivalence relation $\sim$ defined by

$$
x \sim y \Leftrightarrow y=r(\gamma) \text { and } x=s(\gamma) \text { for some } \gamma \in \Gamma .
$$

Accordingly the "values" $\left(F_{x}, \alpha_{x}\right)$ and $\left(F_{y}, \alpha_{y}\right)$, for $x, y \in \Gamma^{(0)}$ related as in (42), are not independent but connected by the bimeasurable $F(\gamma): F_{x} \rightarrow F_{y}$ fulfilling the above definition properties.

The preceding definitions become indispensable in the case of a standard measurable $\Gamma^{(0)}$ with an equivalence relation $\sim$ which is not smooth, so that the quotient $\Gamma^{(0)} / \sim$ is not standard (not even countably separated!). The quotient measurable structure is in that case totally worthless ${ }^{16}$, and in order to integrate over $\Gamma^{(0)} / \sim$, one has in fact to work with a "desingularization" $\Gamma^{(0)}$, keeping track of the necessary compatibility conditions by means of the maps $F_{\gamma}, \gamma \in \Gamma$.

The next Proposition gives a description of the simplest and most useful $\Gamma-\delta$-functions: those arising from the transverse functions $v$ on $\Gamma$.

15 Note that with $\alpha=(\mathbb{F}, \alpha)$ a $\Gamma$ - $\delta$-function, $\alpha^{\prime}=\left(\mathbb{F}^{\prime}, \alpha \circ \varrho\right)$ is a $\Gamma$ - $\delta$-function

16 For instance, in the case of a groupoid $\Gamma$ arising from a group action ergodic and nontransitive (cf. Appendix $\mathrm{B}, 4$ ) the only measurable functions for the quotient measurable structure are the constants 
Proposition 6. Let $\Gamma$ be a standard measurable groupoid and consider the triple $\mathbb{F}_{\Gamma}=(\Gamma, r, L)$, with $\left.L_{x}=\Gamma^{x}=r^{-1}(\{r\}(x)\}\right)$, and $L(\gamma): \Gamma^{x} \rightarrow \Gamma^{y}$ the left multiplication by $\gamma: x \rightarrow y . \mathbb{F}_{\Gamma}$ is a measurable $\Gamma$-functor. Further, with $\delta$ a modular function on $\Gamma$, and $v \in \mathscr{E}^{+},\left(\mathbb{F}_{\Gamma}, \delta^{-1} v\right)=\left(\Gamma, r, L, \delta^{-1} v\right)$ is a $\Gamma$ - $\delta$-function; and for each $\sigma$-finite $\Gamma$-kernel $\lambda$ such that $\lambda\left(\mathbb{1}_{\Gamma}\right)=\mathbb{1}_{\Gamma(0)}, R_{\lambda}: \Gamma \rightarrow \Gamma$ is a natural transformation from $\mathbb{F}_{\Gamma}$ to itself. Finally, given a measurable $\Gamma$-functor $\mathbb{F}=(Z, \pi, F)$, the kernel $\varrho: \Gamma \rightarrow Z$ given by

$$
\left.\begin{array}{ll}
\varrho(f)=f v * u, & f \in \overline{\mathscr{F}}+(\Gamma), \\
u \in \overline{\mathscr{F}}+(Z) \quad \text { such that } \quad v * u=\mathbb{1}_{Z},
\end{array}\right\}
$$

is a natural transformation from $\mathbb{F}$ to $\mathbb{F}_{\Gamma}$.

Proof. The fact that $\mathbb{F}_{\Gamma}$ is a measurable functor readily follows from the definitions; and $\left(\mathbb{F}_{\Gamma}, \delta^{-1} v\right)$ is a $\Gamma$ - $\delta$-function, since the $\Gamma$-kernel $\delta^{-1} v$ is fibered by $r$ and $\operatorname{id}_{\Gamma^{(0)}}$, and we have

$d\left[\left(\delta^{-1} v\right)^{r(\gamma)}\left(\gamma^{\prime}\right)\right]=\delta^{-1}\left(\gamma^{\prime}\right) d v^{r(\gamma)}\left(\gamma^{\prime}\right)=\delta^{-1}(\gamma) \delta^{-1}\left(\gamma^{-1} \gamma^{\prime}\right) d v^{s(\gamma)}\left(\gamma^{-1} \gamma^{\prime}\right)$.

We noted in (10) that $R_{\lambda}: \Gamma \rightarrow \Gamma$ is fibered by $r$ and $r ; R_{\lambda}$ is unital since $R_{\lambda}\left(\mathbb{\mathbb { 1 }}_{\Gamma}\right)$ $=\lambda\left(\mathbb{1}_{\left.\Gamma^{(0)}\right) \circ s}\right.$; and equivariance immediately follows from (6)

$$
R_{\lambda}^{\gamma \gamma^{\prime}}(f)=\int d \lambda^{s\left(\gamma \gamma^{\prime}\right)}\left(\gamma^{\prime \prime}\right) f\left(\gamma \gamma^{\prime} \cdot \gamma^{\prime \prime}\right)=R^{\gamma^{\prime}}(f(\gamma \cdot)) .
$$

The kernel $\varrho: \Gamma \rightarrow Z$ is fibered by $r$ and $\pi$ since, for $\varphi \in \overline{\mathscr{F}}^{+}\left(\Gamma^{(0)}\right)$

$$
\varrho^{z}((\varphi \circ r) f)=\int d v^{\pi(z)}(\gamma) f(\gamma) \varphi(r(\gamma)) u\left(\gamma^{-1} z\right)=\varphi(\pi(z)) \varrho^{z}(f) ;
$$

it is unital since $v * u=\mathbb{1}_{Z}$ and equivariant, since

$$
\begin{aligned}
\varrho^{\gamma z}(f) & =\int d v^{\pi(\gamma z)=r(\gamma)}\left(\gamma^{\prime}\right) f\left(\gamma^{\prime}\right) u\left(\gamma^{\prime-1} \gamma z\right) \\
& =\int d v^{s(\gamma)=\pi(z)}\left(\gamma^{\prime}\right) f\left(\gamma \gamma^{\prime}\right) u\left(\gamma^{\prime-1} z\right) .
\end{aligned}
$$

Proposition 7. Let $\mathbb{F}=(Z, \pi, F)$ be a measurable $\Gamma$-functor. Defining $F_{\lambda} u=\lambda * u$ for $\lambda$ a $\Gamma$-kernel and $u \in \overline{\mathscr{F}}^{+}(Z)$ one obtains, if $\lambda$ is $\sigma$-finite, a kernel $\Gamma_{\lambda}: Z \rightarrow Z$ with the properties

$$
\begin{aligned}
\left(F_{\lambda} u\right)(\gamma z)= & \left(F_{\lambda} u\right)(z), \quad z \in Z, \gamma \in \Gamma \quad \text { such that } \quad s(\gamma)=\pi(z), \\
& F_{\lambda} \circ F_{\mu}=F_{\lambda * \mu}, \lambda, \mu \quad \sigma \text {-finite } \Gamma \text {-kernels. }
\end{aligned}
$$

Furthermore, the following properties of $\mathbb{F}$ are equivalent:

(i) For each faithful $v \in \mathscr{E}^{+}$the kernel $F_{v}: Z \rightarrow Z$ is proper.

(ii) There a faithful $v \in \mathscr{E}^{+}$with $F_{v}$ proper.

(iii) There are $v \in \mathscr{E}^{+}$and $u \in \overline{\mathscr{F}}^{+}(Z)$ with $v * u=\mathbb{\mathbb { 1 }}_{Z}$.

(iv) $\mathbb{F}$ is proper.

Proof. The verification of (52) and (53) is immediate from the definitions. (iii) $\Leftrightarrow$ (iv): $\Leftarrow$ is obvious (assuming as we do the existence of a faithful $v \in \mathscr{E}^{+}$) $\Rightarrow$ : if $v * u=\mathbb{1}_{Z}, v \in \mathscr{E}^{+}, u \in \overline{\mathscr{F}}^{+}(Z)$, and if $v^{\prime} \in \mathscr{E}^{+}$is faithful, there is a $\Gamma$-kernel $\lambda$ such that $v=v^{\prime} * \lambda$ (cf. Proposition 4): then $v^{\prime} * u^{\prime}=\mathbb{1}_{Z}$ for $u^{\prime}=\lambda * u$.

(ii) $\Rightarrow$ (iii): $F_{v}$ is proper whenever there is a never vanishing $v \in \overline{\mathscr{F}}^{+}(Z)$ with $v * v$ 
bounded: the latter accordingly never vanishes, and neither does $u=(v * v)^{-1} v$ : one has $v * u=(v * v)^{-1}(v * v)=\mathbb{1}_{Z}$, as one sees from (40), using (52).

(iii) $\Rightarrow(\mathrm{i})$ : let $v$ and $u$ be as in (iii): there is a $k \in \overline{\mathscr{F}}^{+}(\Gamma)$ never vanishing and such that $v * k=v(\tilde{k}) \circ s=\mathbb{1}_{\Gamma}$. From the fact that $v * u=\mathbb{1}_{Z}$ one sees that $u_{1}=k v * u$ never vanishes since $k$ never vanishes. But $v * u_{1}=v * k v * u=(v * k) v * u=v * u=\mathbb{1}_{Z}$ (cf. Proposition 3). The fact that $F_{\lambda} u$ is measurable as a consequence of the $\sigma$-finiteness of $\lambda$ is proven in Appendix A.

\section{Transverse Measures on a Measurable Groupoid}

We now describe the way in which the $\Gamma-\delta$-functions described above are integrated. With $\Gamma$ a measurable groupoid and $\delta$ a modular function on $\Gamma$ as above, we say that $\Lambda$ is a transverse $\Gamma-\delta$-measure if $\Lambda$ is a map from the $\Gamma$ - $\delta$-functions to $\bar{R}^{+}$ which is

(i) affine, i.e. $\Lambda\left(c \alpha+c^{\prime} \alpha^{\prime}\right)=c \Lambda(\alpha)+c^{\prime} \Lambda\left(\alpha^{\prime}\right), c, c^{\prime} \in R^{+}, \alpha, \alpha^{\prime} \Gamma-\delta$-functions with the same measurable functor $\mathbb{I F}^{17}$,

(ii) normal, i.e. $\Lambda\left(\alpha_{n}\right) \succ \Lambda(\alpha)$ if $\alpha_{n} \succ \alpha, \alpha_{n}$ any ascending sequence of $\Gamma$ - $\delta$ functions with the same $\mathbb{F}^{18}$,

(iii) natural, i.e. $\Lambda(\alpha)=\Lambda\left(\alpha^{\prime}\right)$ whenever $\alpha^{\prime}=\alpha \circ \varrho, \alpha, \alpha^{\prime}$ proper $\Gamma$ - $\delta$-functions with respective measurable functors $\mathbb{F}, \mathbb{F}^{\prime} \varrho$ a natural transformation from $\mathbb{F}$ to $\mathbb{F}^{\prime}$.

The following theorem indicates how one obtains transverse $\Gamma$ - $\delta$-measures.

Theorem 8. Let $\Gamma$ be a measurable groupoid with $\delta$ a modular function on $\Gamma$. Let $v_{0}$ be a faithful transverse function on $\Gamma$ and let $M$ be a positive measure on $\Gamma^{(0)}$ such that $M \circ v_{0}$ is $\delta$-symmetric:

$$
M\left(v_{0}\left(\delta^{-1} \tilde{f}\right)\right)=M\left(v_{0}(f)\right), \quad f \in \overline{\mathscr{F}}^{+}(\Gamma) .
$$

Then, defining, for the $\Gamma$ - $\delta$-function $\alpha=(Z, \pi, F, \alpha)$ :

$$
\Lambda(\alpha)=\operatorname{Sup}\left\{M(\alpha(u)) ; z \in \overline{\mathscr{F}}^{+}(Z), v_{0} * u \leqq \mathbb{1}_{Z}\right\},
$$

we obtain a transverse $\Gamma-\delta$-measure in the above sense. Furthermore one obtains $M$ back from $\Lambda$ as $M=\Lambda_{v_{0}}$ with $^{19}$

$$
\Lambda_{v_{0}}(\varphi)=\Lambda\left(\delta^{-1}(\varphi \circ s) v_{0}\right), \varphi \in \overline{\mathscr{F}}^{+}\left(\Gamma^{(0)}\right) .
$$

For proper $\Gamma$ - $\delta$-functions $\alpha, \Lambda$ can be defined by

$$
\Lambda(\alpha)=M(\alpha(u)), \quad \text { any } \quad u \in \mathscr{F}^{+}(Z) \quad \text { such that } \quad v_{0} * u=\mathbb{1}_{Z} .
$$

The key device for our proof is the following

17 One could equivalently require $\Lambda\left(\alpha \oplus \alpha^{\prime}\right)=\Lambda(\alpha)+\Lambda\left(\alpha^{\prime}\right)$ for general $\Gamma-\delta$-functions $\alpha, \alpha^{\prime}$, with an obvious definition of the direct sum

18 Or else $\Lambda\left(\bigoplus_{n=1}^{\infty} \alpha_{n}\right)=\sum_{n=1}^{\infty} \Lambda\left(\alpha_{n}\right)$

19 We know (Proposition 6) that $\left(\Gamma, \mathrm{r}, \mathrm{L}, \delta^{-1} v_{0}\right)$ is a $\Gamma-\delta$-function. It is readily verified that the same holds with $\delta^{-1} v_{0}$ replaced by $\delta^{-1}(\varphi \circ s) v_{0}$ 
Lemma 9. Let $\Gamma, \delta, v_{0}$ and $M$ be as in Theorem 7, and let $\alpha=(Z, \pi, F, \alpha)$ be $a$ $\Gamma$ - $\delta$-function. We have that

$$
M\left\{\alpha\left(u\left(v_{0} * v\right)\right)\right\}=M\left\{\alpha\left(v\left(v_{0} * u\right)\right)\right\}, u, v \in \overline{\mathscr{F}}+(Z) .
$$

Proof. We have, for $y \in \Gamma^{(0)}$ :

$$
\begin{aligned}
\left.\alpha^{y}\left(u\left(v_{0} * v\right)\right)\right) & =\int d \alpha^{y}(z) u(z) \int d v_{0}^{\pi(z)}(\gamma) v\left(\gamma^{-1} z\right) \\
& =\int d v_{0}^{y}(\gamma) \int d \alpha^{r(\gamma)}(z) u(z) v\left(\gamma^{-1} z\right)
\end{aligned}
$$

i.e.

where

$$
\left.\alpha\left\{u\left(v_{0} * v\right)\right)\right\}=v_{0}\left(u X_{\alpha} v\right)
$$

$$
\left(u X_{\alpha} v\right)(\gamma)=\int d \alpha^{r}\left(\gamma^{\prime}(z) u(z) v\left(\gamma^{-1} z\right), u, v \in \mathscr{F}^{+}(Z) .\right.
$$

But

$$
u X_{\alpha} v=\delta^{-1}\left(v X_{\alpha} u\right)^{\sim}, u, v \in \mathscr{F}^{+}(Z),
$$

as immediately follows from (39):

$$
\left(u X_{\alpha} v\right)(\gamma)=\int \delta^{-1}(\gamma) d \alpha^{s(\gamma)}(z) u(\gamma z) v(z) .
$$

Now (58) immediately follows from (60) and (62), via (54).

Proof of Theorem 8. Let $v \in \overline{\mathscr{F}}^{+}(Z)$ be such that $v_{0} * v=\mathbb{1}_{Z}$ : if $u, u^{\prime} \in \mathscr{F}^{+}(Z)$ are such that $v_{0} * u \leqq v_{0} * u^{\prime}$, one has $M\left(\alpha\left(v\left(v_{0} * u\right)\right) \leqq M\left(\alpha\left(v\left(v_{0} * u^{\prime}\right)\right)\right)\right.$, hence from Lemma $7 M(\alpha(u)) \leqq M\left(\alpha\left(u^{\prime}\right)\right)$. This shows that $\Lambda$ is given by (57) for proper $\Gamma-\delta$ functions $\alpha$. For the latter we could define $\Lambda$ in this way, Lemma 7 making it obvious that the expression on the right-hand side of (57) does not depend from $u$ subject to $v_{0} * v_{u}=\mathbb{1}_{Z}$. The facts that $\Lambda$ is affine and normal follow from these properties for the map $\alpha \rightarrow M(\alpha(u))$ for a fixed $u$. And naturality follows readily from (57) and (44): let $\alpha$ and $\alpha^{\prime}=\alpha \circ \varrho$ be proper $\Gamma$ - $\delta$-functions related by the natural transformation $\varrho$ and let $u^{\prime} \in \overline{\mathscr{F}^{+}}\left(Z^{\prime}\right)$ be such that $v_{0} * u^{\prime}=\mathbb{1}_{Z^{\prime}}$ : we have

$$
\Lambda(\alpha \circ \varrho)=M\left(\alpha\left(\varrho\left(u^{\prime}\right)\right)\right)=\Lambda(\alpha),
$$

since $\varrho\left(u^{\prime}\right)$ is such that

$$
v_{0} * \varrho\left(u^{\prime}\right)=\varrho\left(v_{0} * u^{\prime}\right)=v\left(\mathbb{1}_{Z^{\prime}}\right)=\mathbb{1}_{Z} .
$$

Next, the fact that $M=\Lambda_{v}$ follows from (57) applied to $\alpha=\delta^{-1}(\varphi \circ s) v_{0}$ using the $\delta$-symmetry of $M \circ v_{0}$ :

$$
\begin{aligned}
\Lambda\left(\delta^{-1}(\varphi \circ s) v_{0}\right) & =M\left(\delta^{-1}(\varphi \circ s) v_{0}(f)\right), f \in \overline{\mathscr{F}}^{+}(\Gamma) \text { such that } v_{0} * f=\mathbb{1}_{\Gamma} \\
& =M\left(v_{0}\left(\delta^{-1}(\varphi \circ s) f\right)\right. \\
& =M\left(v_{0}((\varphi \circ r) \tilde{f})\right)=M\left(\varphi v_{0}(\tilde{f})\right)=M(\varphi) .
\end{aligned}
$$

We now prove the related facts that $\Gamma$ - $\delta$-measures are determined by their restrictions to the $\Gamma$ - $\delta$-functions $\delta^{-1} v$ of Proposition 6; and that the construction method of Theorem 8 yields in fact the most general $\Lambda$, whereby $v_{0}$ can be taken as any faithful transverse function. 
Theorem 10. Fix a modular function $\delta$ on the measurable groupoid $\Gamma$ and set ${ }^{20}$

$$
\Lambda^{\prime}(v)=\Lambda\left(\delta^{-1} v\right), v \in \mathscr{E}^{+},
$$

one thus gets a bijection between the $\Gamma$ - $\delta$-measures $\Lambda$ and the maps $\Lambda^{\prime}: \mathscr{E}^{+} \rightarrow \bar{R}^{+}$ which are

(i) affine,

(ii) normal,

(iii) fulfilling one of the two equivalent following conditions (68) or (69):

$$
\left.\begin{array}{c}
\Lambda^{\prime}(v * \lambda)=\Lambda^{\prime}(v) \text { for all } v \in \mathscr{E}^{+} \text {and } \sigma \text {-finite } \\
\Gamma \text {-kernels } \lambda \text { with } \lambda\left(\delta^{-1}\right)=\mathbb{1}_{\Gamma^{(0)}}, \\
\Lambda^{\prime}\left(v * \lambda_{1}\right)=\Lambda^{\prime}\left(v * \lambda_{2}\right) \text { for all } v \in \mathscr{E}^{+} \text {and } \sigma \text {-finite } \\
\Gamma \text {-kernels } \lambda_{1}, \lambda_{2} \text { with } \lambda_{1}\left(\delta^{-1}\right)=\lambda_{2}\left(\delta^{-1}\right) .
\end{array}\right\}
$$

Fix furthermore an arbitrary faithful $v_{0} \in \mathscr{E}^{+}$and define $\Lambda_{v_{0}}$ as in (56): this establishes a bijection between the $\Gamma$ - $\delta$-measures $\Lambda$ and the positive measures $\Lambda_{v_{0}}$ on $\Gamma^{(0)} \delta$-symmetric in the sense of (30). One has

$$
\left.\begin{array}{l}
\Lambda\left(\delta^{-1}\left(v_{0} * \lambda\right)\right)=\Lambda^{\prime}\left(v_{0} * \lambda\right)=\Lambda_{v_{0}}\left(\lambda\left(\delta^{-1}\right)\right), \\
v_{0} \in \mathscr{E}^{+}, \lambda \text { a } \sigma \text {-finite } \Gamma \text {-kernel, }
\end{array}\right\}
$$

and $\Lambda$ is obtained back from $\Lambda_{v_{0}}$ as in (55) and (57) with $M=\Lambda_{v_{0}}$. In particular

$$
\Lambda^{\prime}(v)=\Lambda_{v_{0}}\left(v\left(\delta^{-1} f\right)\right), \quad \text { any } \quad f \in \overline{\mathscr{F}}^{+}(\Gamma) \quad \text { with } \quad v_{0} * f=\mathbb{1}_{\Gamma} .
$$

Proof. Remembering (12) and the fact (cf. Proposition 6) that $R_{\lambda^{\prime}}, \lambda^{\prime}\left(\mathbb{1}_{\Gamma}\right)=\mathbb{1}_{\Gamma^{(0)}}$ is a natural transformation from $\mathbb{1}$ to itself, we have that

$$
\Lambda\left(\delta^{-1} v * \lambda^{\prime}\right)=\Lambda\left(\delta^{-1} v\right), \quad\left\{\begin{array}{l}
\lambda^{\prime} \text { a } \sigma \text {-finite } \Gamma \text {-kernel with } \\
\lambda^{\prime}\left(\mathbb{1}_{\Gamma}\right)=\mathbb{1}_{\Gamma^{(0)}}, v \in \mathscr{E}^{+}
\end{array}\right.
$$

The transcription of this for the notation (67) yields property (68) taking account of the straightforward facts that each modular function $\delta$ on $\Gamma$ fulfills

$$
\left.\left.\begin{array}{l}
\delta(\lambda * f)=\delta \lambda * \delta f, \\
\delta(\lambda * \mu)=\delta \lambda * \delta \mu,
\end{array}\right\} \begin{array}{l}
f \in \overline{\mathscr{F}}^{+}(\Gamma), \\
\lambda, \mu \sigma \text {-finite } \Gamma \text {-kernels. }
\end{array}\right\}
$$

For the rest of our proof we need

Lemma 11. Setting

$\left(\varepsilon_{\varphi}\right)^{y}=\varphi(y) \varepsilon_{y}, \quad y \in \Gamma^{(0)}, \varepsilon_{y} \quad$ the Dirac measure at $y$, yields a linear map $\varphi \rightarrow \varepsilon_{\varphi}$ from the finite elements $\varphi$ of $\overline{\mathscr{F}}+\left(\Gamma^{(0)}\right)$ to $\sigma$-finite $\Gamma$-kernels $\varepsilon_{\varphi}$. This map turns the pointwise product into convolution and one has

(i) $\varepsilon_{\varphi} * f=(\varphi \circ r) f, \quad f \in \overline{\mathscr{F}}^{+}(\Gamma)$,

(ii) $\left\{\begin{array}{l}\varepsilon_{\varphi} * \lambda=(\varphi \circ r) \lambda \\ \lambda * \varepsilon_{\varphi}=(\varphi \circ s) \lambda\end{array}, \quad \lambda\right.$ a $\sigma$-finite $\Gamma$-kernel,

(iii) each $\Gamma$-kernel $\lambda$ with $\lambda\left(\delta^{-1}\right)$ finite can be written as $\varepsilon_{\lambda\left(\delta^{-1}\right)} * \mu, \mu$ a $\Gamma$-kernel with $\mu\left(\delta^{-1}\right)=\mathbb{1}_{\Gamma^{(0)}}$. 
Proof of Lemma. (i) follows straightforwardly from (74) and (4); (ii) follows from the fact that, for each $f \in \overline{\mathscr{F}}^{+}(\Gamma)$

$$
\left.\begin{array}{l}
\left(\varepsilon_{\varphi} * \lambda\right)(f)=\left.\varepsilon_{\varphi} * \lambda * \tilde{f}\right|_{\Gamma^{(0)}}=\left.[(\varphi \circ r)(\lambda * \tilde{f})]\right|_{\Gamma^{(0)}}=\varphi \lambda(f)=\lambda((\varphi \circ r) f), \\
\left(\lambda * \varepsilon_{\varphi}\right)(f)=\left.\lambda * \varepsilon_{\varphi} * \tilde{f}\right|_{\Gamma^{(0)}}=\lambda\left(\left(\varepsilon_{\varphi} * \widetilde{f}\right)^{\sim}\right)=\lambda\left[((\varphi \circ r) \tilde{f})^{\sim}\right]=\lambda((\varphi \circ s) f) .
\end{array}\right\}
$$

For $\varphi, \varphi^{\prime} \in \overline{\mathscr{F}}^{+}\left(\Gamma^{(0)}\right)$ finite the fact that $\varepsilon_{\varphi \varphi^{\prime}}=\varepsilon_{\varphi} * \varepsilon_{\varphi^{\prime}}$ then results from

$$
\varepsilon_{\varphi} * \varepsilon_{\varphi^{\prime}} * f=\varepsilon_{\varphi} *\left(\left(\varphi^{\prime} \circ r\right) f\right)=(\varphi \circ r)\left(\varphi^{\prime} \circ r\right) f=\left(\varphi \varphi^{\prime} \circ r\right) f, \quad f \in \overline{\mathscr{F}}^{+}(\Gamma) .
$$

We now prove (iii): consider a $\sigma$-finite $\Gamma$-kernel $\lambda$ with $\lambda\left(\delta^{-1}\right)$ finite and let $S_{\lambda}$, with complement $S_{\lambda}^{\prime}$, be as defined in (15). Define $\varphi \in \overline{\mathscr{F}}+\left(\Gamma^{(0)}\right)$ by $\varphi(y)=\lambda^{(y)}\left(\delta^{-1}\right)^{-1}, y \in S_{\lambda}$ and $\varphi(y)=0, y \in S_{\lambda}^{\prime}$ and let $\mu=\varepsilon_{\varphi} * \lambda+\varepsilon_{\mathbb{1}_{S^{\prime}}}$ : we have $\mu\left(\delta^{-1}\right)=\varphi \lambda\left(\delta^{-1}\right)+\mathbb{1}_{S_{\lambda}^{\prime}}=\mathbb{1}_{S_{\lambda}}+\mathbb{1}_{S^{\prime}}=\mathbb{1}_{\Gamma^{(0)}}$; and $\varepsilon_{\lambda\left(\delta^{-1}\right)} * \mu=\varepsilon_{\lambda\left(\delta^{-1}\right) \varphi} * \lambda=\left(\mathbb{1}_{S_{\lambda}} \circ r\right) \lambda=\lambda$.

Proof of End of Theorem. Proof of $(68) \Leftrightarrow(69): \Leftarrow$ follows from taking $\lambda_{1}=\lambda$ and $\lambda_{2}$ $=\varepsilon_{\mathbb{1}_{\Gamma^{(0)}}}$ in $(69) ; \Rightarrow$ follows from the relation

$$
\Lambda^{\prime}(v * \lambda)=\Lambda^{\prime}\left(v * \varepsilon_{\lambda\left(\delta^{-1}\right)}\right)=\Lambda\left(\left(\lambda\left(\delta^{-1}\right) \circ s\right) v\right), \quad v \in \mathscr{E}^{+}
$$

resulting from Lemma 11 (iii) and (68) in the case of a finite $\lambda\left(\delta^{-1}\right)$ (observe that $\left.\varepsilon_{\lambda\left(\delta^{-1}\right)}\left(\delta^{-1}\right)=\lambda\left(\delta^{-1}\right)\right)$; and persisting for general $\sigma$-finite kernels $\lambda$ as one sees approximating the latter by ascending sequences $\left\{\lambda_{n}\right\}$ for which the $\lambda_{n}\left(\delta^{-1}\right)$ are finite. Note that with (77) we proved (70). The $\delta$-symmetry of $\Lambda_{v_{0}} \circ v_{0}$ now readily follows: one has since $\left(v_{0}(f) \circ s\right) v=\left(v_{0} * \tilde{f}\right) v_{0}=v_{0} * \tilde{f} \tilde{v}_{0}$,

$$
\Lambda_{v_{0}}\left(v_{0}(f)\right)=\Lambda^{\prime}\left(\left(v_{0}(f) \circ s\right) v\right)=\Lambda^{\prime}\left(v_{0} * \tilde{f v_{0}}\right)=\Lambda_{v}\left(\delta^{-1} v_{0}(\tilde{f})\right) .
$$

It remains to show (57) with $M=\Lambda_{v_{0}}$. For this we recall that $f \in \overline{\mathscr{F}}^{+}(\Gamma) \rightarrow v_{0} f * u$, $u \in \overline{\mathscr{F}}^{+}(Z)$ with $v_{0} * u=\mathbb{1}_{Z}$, is a natural transformation from $\mathbb{F}_{\text {to }} \mathbb{F}_{\Gamma}$. Let $v=\alpha \circ \varrho$ : by the naturality of $\Lambda$, we have $\Lambda(\alpha)=\Lambda(v)$. We calculate $v$ using the fact that we have, for each $\Gamma$ - $\delta$-function $\alpha=(Z, \pi, F, \alpha)$

$$
\left.\alpha(\lambda * u)=\left(\delta^{-1} \lambda\right)(\alpha(u) \circ s)\right), \quad u \in \overline{\mathscr{F}}^{+}(Z), \quad \lambda \text { a } \Gamma \text {-kernel, }
$$

indeed:

$$
\begin{aligned}
\alpha^{y}(\lambda * u) & =\int d \alpha^{y}(z) \int d \lambda^{\pi(z)}(\gamma) u\left(\gamma^{-1} z\right)=\int d \lambda^{y}(\gamma) \int d \alpha^{r(\gamma)}(z) u\left(\gamma^{-1} z\right) \\
& =\int d \lambda^{y}(\gamma) \delta^{-1}(\gamma) \int d \alpha^{s(\gamma)}(z) u(z) .
\end{aligned}
$$

Now

thus

$$
v(f)=\alpha\left(f v_{0} * u\right)=\delta^{-1} f v_{0}(\alpha(u) \circ s)=\delta^{-1}(\alpha(u) \circ s) v_{0}(f),
$$

$$
\Lambda(\alpha)=\Lambda(v)=\Lambda\left(\delta^{-1}(\alpha(u) \circ s) v_{0}\right)=\Lambda_{v_{0}}(\alpha(u)) .
$$

\section{Transport of Transverse Measures by Groupoid Homomorphisms}

Let $\Gamma$ and $\Gamma^{\prime}$ be measurable groupoids. A homomorphism from $\Gamma$ to $\Gamma^{\prime}$ is a measurable map $h: \Gamma \rightarrow \Gamma^{\prime}$ such that

$$
h\left(\gamma^{-1} \gamma^{\prime}\right)=h(\gamma)^{-1} h\left(\gamma^{\prime}\right), \gamma, \gamma^{\prime} \in \Gamma, r(\gamma)=r\left(\gamma^{\prime}\right) .
$$

It follows immediately that $h$ conserves products and inverses; that $h\left(\Gamma^{(0)}\right) \subset \Gamma^{\prime(0)}$; and that $h \circ r=r^{\prime} \circ h, h \circ s=s^{\prime} \circ h$ : in other terms $\gamma: x \rightarrow y$ implies $h(\gamma): h(x) \rightarrow h(y)$. 
Note that the groupoid homomorphisms are the endofunctors of the category of measurable groupoids. Two homomorphisms $h, h^{\prime}: \Gamma \rightarrow \Gamma^{\prime}$ are called conjugate (or similar) if they are connected by a natural transformation, i.e., if there is a measurable map $\theta: \Gamma^{(0)} \rightarrow \Gamma^{\prime}$ such that one has

$$
\left.\begin{array}{l}
\theta(x): h(x) \rightarrow h^{\prime}(x), x \in \Gamma^{(0)} \\
\theta(y) \circ h(\gamma)=h^{\prime}(\gamma) \circ \theta(x), \gamma \in \Gamma, \gamma: x \rightarrow y .
\end{array}\right\}
$$

With $h: \Gamma \rightarrow \Gamma^{\prime}$ a groupoid homomorphism, and respective modular functions $\delta, \delta^{\prime}$ on $\Gamma, \Gamma^{\prime}$ such that $\delta^{\prime} \circ h=\delta$, we shall now define the transport by $h$ of transverse $\Gamma$ - $\delta$-measures. This is effected, analogously to the case of usual measures, by first defining the pull-back of $\Gamma$ - $\delta$-functions. Since naturality is part of the definition of transverse measures, we shall also need to define the pull-back of natural transformations.

Let thus $\alpha^{\prime}=\left(\mathbb{F}^{\prime}, A^{\prime}\right)=\left(Z^{\prime}, \pi^{\prime}, F^{\prime}, \alpha^{\prime}\right)$ be a $\Gamma$ - $\delta$-function: its pull-back $h^{*} \alpha^{\prime}=\alpha=(\mathbb{F}, \alpha)=(Z, \pi, F, \alpha)$ is defined as follows: the measurable $\Gamma$-functor $\mathbb{F}$ is given by

with

$$
Z=\left\{\left(z^{\prime}, x\right) \in Z^{\prime} \times \Gamma^{(0)} ; \pi^{\prime}\left(z^{\prime}\right)=h(x)\right\},
$$

$$
\left.\begin{array}{ll}
\pi\left(z^{\prime}, x\right)=x, & x, y \in \Gamma^{(0)}, \\
F_{x}=F_{h(x)}^{\prime}, & z^{\prime} \in Z^{\prime}, \pi\left(z^{\prime}\right)=h(x), \\
F(\gamma)\left(z^{\prime}, x\right)=\left(h(\gamma) z^{\prime}, y\right), & \gamma \in \Gamma, \gamma: x \rightarrow y,
\end{array}\right\}
$$

whilst the kernel $\alpha=h^{*} \alpha^{\prime}: Z \rightarrow \Gamma^{(0)}$ is given by

$$
\begin{aligned}
\left(h^{*} \alpha^{\prime}\right)^{x}(u) & =\alpha^{\prime h(x)}(u(\cdot, x)) \\
& =\int d \alpha^{\prime h(x)}\left(z^{\prime}\right) u\left(z^{\prime}, x\right)^{\prime} \quad u \in \overline{\mathscr{F}}^{+}(Z), x \in \Gamma^{(0)} .
\end{aligned}
$$

Let now $\varrho^{\prime}: Z_{1}^{\prime} \rightarrow Z^{\prime}$ be a natural transformation from $\mathbb{F}^{\prime}$ to $\mathbb{F}_{1}^{\prime}, \mathbb{F}_{1}^{\prime}$ $=\left(Z_{1}^{\prime}, \pi_{1}^{\prime}, F_{1}^{\prime}\right)$. With $\mathbb{F}, \mathbb{F}_{1}$ the pull-backs of $\mathbb{F}^{\prime}, \mathbb{F}_{1}^{\prime}$ defined as above, the pull-back $\varrho=h^{*} \varrho^{\prime}$ of $\varrho^{\prime}$ is defined as

i.e.

$$
\begin{aligned}
\left(h^{*} \varrho^{\prime}\right)^{\left(z^{\prime}, x\right)}(u) & =\varrho^{\prime} z^{\prime}\left(u_{1}(\cdot, x)\right) \\
& =\int d \varrho^{\prime z^{\prime}}\left(u_{1}^{\prime}\right) u_{1}\left(z_{1}^{\prime}, x\right), \quad\left(z^{\prime}, x\right) \in Z, u_{1} \in \overline{\mathscr{F}}^{+}\left(Z_{1}\right),
\end{aligned}
$$

$$
\left(h^{*} \varrho^{\prime}\right)\left(u_{1}\right)(\cdot, x)=\varrho^{\prime}\left(u_{1}(\cdot, x)\right), u_{1} \in \overline{\mathscr{F}}+\left(Z_{1}\right) .
$$

Finally, the image by $h$ of the transverse $\Gamma-\delta$-measure $\Lambda$ is the $\Gamma^{\prime}-\delta^{\prime}$-measure $h \Lambda$ defined as

$$
(h \Lambda)\left(\alpha^{\prime}\right)=\Lambda\left(h^{*} \alpha^{\prime}\right), \alpha^{\prime} \text { any } \Gamma^{\prime}-\delta^{\prime} \text {-function. }
$$

We check the legitimacy of the above definitions. $Z$ is measurable in $Z^{\prime} \times \Gamma^{(0)}$ (thus standard) as the preimage of the diagonal in $\Gamma^{(0)} \times \Gamma^{(0)}$ for the map $\pi^{\prime} \times h$. $F_{x}=F_{h(x)}^{\prime}$ is isomorphic to $\pi^{-1}(x) \sim \pi^{\prime-1}(h(x))$; and $F(\gamma): \Gamma \times Z \rightarrow Z$, clearly measurable, is such that

$$
\begin{aligned}
F\left(\gamma^{\prime-1} \gamma\right)\left(z^{\prime}, x\right) & =\left(\gamma^{\prime-1} \gamma z^{\prime}, u\right)=F\left(\gamma^{\prime-1}\right)\left(\gamma z^{\prime}, y\right) \\
& =F\left(\gamma^{\prime-1}\right) F(\gamma)\left(z^{\prime}, x\right),
\end{aligned} \quad\left\{\begin{array}{l}
\gamma, \gamma^{\prime} \in \Gamma \\
\gamma: x \rightarrow y, \gamma^{\prime}: u \rightarrow y
\end{array}\right.
$$


Further $\alpha=h * \alpha^{\prime}$, clearly a kernel: $Z \rightarrow \Gamma^{(0)}$, is fibered by $\pi$ and $\operatorname{id}_{\left.\Gamma^{(0)}\right)}$ :

$$
\left.\alpha^{\prime h(x)}([(\varphi \circ \pi) u](\cdot, x))=\varphi(x) \alpha^{\prime h(x)}(\cdot, x)\right), \varphi \in \overline{\mathscr{F}}^{+}\left(\Gamma^{(0)}\right) ;
$$

and equivariant: writing as usual $\Gamma(\gamma)=\gamma$ for shortness

$$
\begin{aligned}
\left(\gamma \alpha^{x}\right)(u)=\alpha^{x}(u \circ \gamma) & =\int d \alpha^{\prime h(x)}\left(z^{\prime}\right) u\left(h(\gamma) z^{\prime}, y\right) \\
& =\int d \alpha^{\prime h(y)}\left(z^{\prime}\right) \delta^{\prime}(h(\gamma)) u^{\prime}\left(z^{\prime}, y\right), \quad \gamma: x \rightarrow y
\end{aligned}
$$

whence $\gamma \alpha^{x}=\delta(\gamma) \alpha^{y}, \gamma: x \rightarrow y$, since $\delta^{\prime}(h(\gamma))=\delta(\gamma)$.

We now check that $\varrho=h^{*} \varrho^{\prime}$ is a natural transformation: $\varrho$ is clearly a kernel: $Z_{1} \rightarrow Z$, it is fibered by $\pi_{1}$ and $\pi$ :

$$
\varrho^{\prime}\left(\left[\left(\varphi \circ \pi_{1}\right)\left(u_{1}\right)(\cdot, x)\right)=\varphi(x) \varrho^{\prime}\left(u_{1}(\cdot, x)\right), \varphi \in \overline{\mathscr{F}}^{+}\left(\Gamma^{(0)}\right),\right.
$$

equivariant:

$$
\begin{aligned}
\gamma \varrho^{\left(z^{\prime}, x\right)}\left(u_{1}\right) & =\varrho^{\left(z^{\prime}, x\right)}\left(u_{1} \circ \gamma\right)=\varrho^{\prime z^{\prime}}\left(\left(u_{1} \circ \gamma\right)(\cdot, x)\right) \\
& =\varrho^{\prime z^{\prime}}\left(u_{1}(h(\gamma) \cdot, y)\right)=\left(h(\gamma) \varrho^{\prime z^{\prime}}\right)\left(u_{1}(\cdot, y)\right) \\
& =\varrho^{\prime h(\gamma) z^{\prime}}\left(u_{1}(\cdot, y)\right)=\varrho^{\gamma(z \prime, x)}\left(u_{1}\right),
\end{aligned}
$$

and unital:

$$
\left(\varrho \mathbb{1}_{Z_{1}}\right)(\cdot, x)=\varrho^{\prime}\left(\mathbb{1}_{Z_{1}}(\cdot, x)\right)=\left(\varrho^{\prime} \mathbb{1}_{Z_{1}^{\prime}}\right)(\cdot)=\mathbb{1}_{Z^{\prime}}(\cdot) .
$$

We note that with the preceding definitions we have, as expected, the fact that

indeed

$$
h^{*}\left(\alpha^{\prime} \varrho^{\prime}\right)=\left(h^{*} \alpha^{\prime}\right)\left(h^{*} \varrho^{\prime}\right),
$$

$$
h^{*}\left(\alpha^{\prime} \varrho^{\prime}\right)^{x}\left(u_{1}\right)=\left(\alpha^{\prime} \varrho^{\prime}\right)^{h(x)}\left(u_{1}(\cdot, x)\right)=\alpha^{\prime h(x)}\left(\varrho^{\prime}\left(u_{1}(\cdot, x)\right)\right) \text {. }
$$

We now check that (90) yields a transverse $\Gamma^{\prime}-\delta^{\prime}$-function. The fact that $h \Lambda$ is affine and normal is straightforward. In order to check naturality we need

Lemma 12. Let the homomorphism $h: \Gamma \rightarrow \Gamma^{\prime}$ be proper in the sense that the functor $h * \mathbb{F}_{\Gamma^{\prime}}$ is proper $\left(\mathbb{F}_{\Gamma^{\prime}}=\left(\Gamma^{\prime}, r^{\prime}, L^{\prime}\right)\right.$ cf. Proposition 6$)$. Then $h * \mathbb{F}^{\prime}$ is proper for every proper $\mathbb{F}^{\prime}$. Consequently, the composition of proper homomorphisms is proper.

Proof of the Lemma. Properness of $h * \mathbb{F}_{\Gamma^{\prime}}$, respectively $\mathbb{F}^{\prime}$ is the existence of functions $g \in \overline{\mathscr{F}}^{+}\left(\Gamma^{\prime} \times \Gamma^{(0)}\right)$, respectively $u^{\prime} \in \overline{\mathscr{F}}^{+}\left(Z^{\prime}\right)$ such that

respectively

$$
(v * g)\left(\gamma^{\prime}, y\right)=\int d v^{y}(\gamma) g\left(h(\gamma)^{-1} \gamma^{\prime}, s(\gamma)\right)=1, \quad\left\{\begin{array}{l}
\gamma^{\prime} \in \Gamma^{\prime}, y \in \Gamma^{(0)} \\
\pi^{\prime}\left(\gamma^{\prime}\right)=h(y),
\end{array}\right.
$$

$$
\left(v^{\prime} * u^{\prime}\right)\left(z^{\prime}\right)=\int d v^{\prime \pi\left(z^{\prime}\right)}\left(\gamma^{\prime}\right) u^{\prime}\left(\gamma^{\prime-1} z^{\prime}\right)=1, z^{\prime} \in Z^{\prime},
$$

for some faithful $v \in \mathscr{E}^{+}, v^{\prime} \in \mathscr{E}^{\prime+}$. We need to exhibit a function $u \in \overline{\mathscr{F}}^{+}(Z)$ with $v * u=\mathbb{1}_{Z}$. Let

$$
u\left(z^{\prime}, x\right)=\int d v^{\prime h(x)}\left(\gamma^{\prime}\right) g\left(\gamma^{\prime}, x\right) u^{\prime}\left(\gamma^{\prime-1} z^{\prime}\right), \quad\left\{\begin{array}{l}
z^{\prime} \in Z^{\prime}, x \in \Gamma^{(0)} \\
\pi^{\prime}\left(z^{\prime}\right)=h(x),
\end{array}\right.
$$


we have, using the invariance of $v^{\prime}$, by (99) and (100)

$$
\begin{aligned}
(v * u)\left(z^{\prime}, y\right) & =\int d v^{\pi\left(z^{\prime}, y\right)}\left(\gamma_{1}\right) u\left(h\left(\gamma_{1}\right)^{-1} z^{\prime}, s\left(\gamma_{1}\right)\right) \\
& =\int d v^{y}\left(\gamma_{1}\right) d v^{\prime h\left(s\left(\gamma_{1}\right)\right)}\left(\gamma^{\prime}\right) g\left(\gamma^{\prime}, s\left(\gamma_{1}\right)\right) u^{\prime}\left(\gamma^{\prime-1} h\left(\gamma_{1}\right)^{-1} z^{\prime}\right) \\
& =\int d v^{y}\left(\gamma_{1}\right) d v^{\prime} h\left(r\left(\gamma_{1}\right)=y\right)\left(\gamma^{\prime}\right) g\left(h\left(\gamma_{1}\right)^{-1} \gamma^{\prime}, s\left(\gamma_{1}\right)\right) u^{\prime}\left(\gamma^{\prime-1} z^{\prime}\right) \\
& =\int d v^{\prime} h(y)\left(\gamma^{\prime}\right) u^{\prime}\left(\gamma^{\prime-1} z^{\prime}\right)=\mathbb{1}_{z} .
\end{aligned}
$$

We proved that $h * \mathbb{F}^{\prime}$ is proper. Now if $h: \Gamma \rightarrow \Gamma^{\prime}$ and $h^{\prime}: \Gamma^{\prime} \rightarrow \Gamma^{\prime \prime}$ are proper, $h * \mathbb{F}_{\Gamma^{\prime}}$ is proper, and so is $\left(h^{\prime} \circ h\right) * \mathbb{F}_{\Gamma^{\prime \prime}}=h\left(h^{\prime} * \mathbb{F}_{\Gamma^{\prime \prime}}\right)$ by what precedes.

We now check naturality of $h \Lambda$. For $\alpha^{\prime}$ a proper $\Gamma^{\prime}$ - $\delta$-function and $\varrho^{\prime}$ a natural transformation as above, we have by (97), since $h * \alpha^{\prime}$ is proper

$$
\begin{aligned}
(h \Lambda)\left(\alpha^{\prime} \varrho^{\prime}\right) & =\Lambda\left(h^{*}\left(\alpha^{\prime} \varrho^{\prime}\right)\right)=\Lambda\left(\left(h^{*} \alpha^{\prime}\right) h^{*} \varrho^{\prime}\right) \\
& =\Lambda\left(h^{*} \alpha^{\prime}\right)=(h \Lambda)\left(\alpha^{\prime}\right) .
\end{aligned}
$$

We proved

Theorem 13. Let $h: \Gamma \rightarrow \Gamma^{\prime}$ be a proper groupoid homomorphism such that $\delta^{\prime} \circ h=\delta, \delta, \delta^{\prime}$ respective modular functions on $\Gamma$ and $\Gamma^{\prime}$. For each transverse $\Gamma$ - $\delta$-measure $\Lambda(90)$ defines a transverse $\Gamma^{\prime}$ - $\delta^{\prime}$-measure $h \Lambda$.

\section{Appendix A}

This appendix supplies the proof of the claim in Proposition 7 that $F_{\lambda}$ is a kernel from $Z$ to $Z$. The fact claimed in Proposition 1 that $L_{\lambda}$ [and consequently $R_{\lambda}$, cf. (6)] are kernels from $\Gamma$ to $\Gamma$ is a special case. The missing fact which we have to prove is that for $\lambda$ a $\sigma$-finite $\Gamma$-kernel and $u \in \overline{\mathscr{F}}^{+}(Z)$ the function $\lambda * u$ defined in (40) is measurable. For the proof we need

Theorem A1. Let $X_{i}=\left(X_{i}, \mathbb{B}_{i}\right), X_{i}^{\prime}=\left(X_{i}^{\prime}, \mathbb{B}_{i}^{\prime}\right)$ be measurable spaces with respective tribes of measurable sets $\mathbb{B}_{i}, \mathbb{B}$ and let $\lambda_{i}: X_{i} \rightarrow X_{i}^{\prime}$ be a $\sigma$-finite kernel, $i=1,2$. Let $X_{1} \times X_{2}$ and $X_{1}^{\prime} \times X_{2}^{\prime}$ be given the product measurable structures ${ }^{21}$ : there is a unique kernel $\lambda_{1} \otimes \lambda_{2}: X_{1} \times X_{2} \rightarrow X_{1}^{\prime} \times X_{2}^{\prime}$ such that

$$
\left(\lambda_{1} \otimes \lambda_{2}\right)\left(B_{1} \times B_{2}\right)=\lambda_{1}\left(B_{1}\right) \lambda_{2}\left(B_{2}\right), B_{1} \in \mathbb{B}_{1}, B_{2} \in \mathbb{B}_{2} \text {. }
$$

Furthermore $\lambda_{1} \otimes \lambda_{2}$ is $\sigma$-finite.

The proof is a modification of the proof of the usual Fubini Theorem. Since $\lambda_{i}$ is $\sigma$-finite, the measure $\lambda_{i}^{x^{\prime}}=\lambda_{i}\left(x^{\prime}, \cdot\right)$ is in particular $\sigma$-finite for each $x^{\prime} \in X_{i}^{\prime}, i=1,2$ : the Fubini Theorem thus entails the existence of a $\sigma$-finite measure $\lambda_{1}^{x_{1}^{\prime}} \otimes \lambda_{2}^{x_{2}}$ on $X_{1} \times X_{2}$ for all $x_{1}^{\prime} \in X_{1}^{\prime}, x_{2}^{\prime} \in X_{2}^{\prime}$. It follows from (A1) that one must have

$$
\left(\lambda_{1} \otimes \lambda_{2}\right)\left(\left(x_{1}^{\prime}, x_{2}^{\prime}\right), S\right)=\left(\lambda_{1}^{x_{1}} \otimes \lambda_{2}^{x^{\prime}}\right)(S), \quad S \in \mathbb{B}_{1} \times \mathbb{B}_{2},
$$

and all we have to check is that the right hand side defines a measurable function on $X_{1}^{\prime} \times X_{2}^{\prime}$ for all $S \in \mathbb{B}_{1} \times \mathbb{B}_{2}$. Let $\Omega$ be set of $S$ for which this is the case. Taking

21 We recall that the tribe $\mathbb{B}_{1} \times \mathrm{B}_{2}$ of measurable sets of $X_{1} \times X_{2}$ is defined as the smallest tribe containing all rectangles $B_{1} \times B_{2}, B_{1} \in \mathbb{B}_{1}, B_{2} \in \mathbb{B}_{2}$ 
account of the fact that

$$
\left(\lambda_{1}^{x_{1}^{\prime}} \otimes \lambda_{2}^{x^{\prime}}\right)(S)=\int d \lambda_{1}^{x_{1}^{\prime}}\left(x_{1}\right) \lambda_{2}^{x^{\prime}}\left(S_{x_{1}}\right)
$$

where $S_{x_{1}}=\left\{x_{2} \in X_{2} ;\left(x_{1}, x_{2}\right) \in S\right\}$, one has that

(a) $\Omega$ contains all measurable rectangles $B_{1} \times B_{2}, B_{1} \in \mathbb{B}_{1}, B_{2} \in \mathbb{B}_{2}$.

(b) $\Omega$ is closed for finite disjoint unions.

(c) $\Omega$ is closed for increasing sequences.

(d) $\Omega$ is closed for countable disjoint unions.

(e) $\Omega$ is closed for decreasing sequences dominated by measurable rectangles $A_{1} \times A_{2}$ such that $\lambda_{i^{\prime}}^{x^{\prime}}\left(A_{i}\right)<\infty, x_{i}^{\prime} \in X_{i}^{\prime}, i=1,2$.

Properties (a)-(c) are immediate from (A3); (b) and (c) imply (d); and (e) follows from the dominated convergence theorem: if the sequence $\left\{S_{k}\right\} \in \Omega$ is such that $S_{k}>S$ with $S_{k} \in A_{1} \times A_{2}$ for all $k$, one has for all $x_{2}^{\prime} \in X_{2}^{\prime}, x_{1} \in X_{1}$ $\lambda_{2}^{x^{\prime}}\left(S_{i_{x_{1}}}\right) \rightarrow \lambda_{2}^{x^{\prime}}\left(S_{x_{1}}\right) \leqq \mathbb{1}_{A}\left(x_{1}\right) \lambda_{2}^{x^{\prime}}(B)$. Hence

$$
\int d \lambda_{1}^{x_{1}^{\prime}}\left(x_{1}\right) \lambda_{2}^{x^{\prime}}\left(S_{i_{x_{1}}}\right) \rightarrow \int d \lambda_{1}^{x_{1}^{\prime}}\left(x_{1}\right) \lambda_{2}^{x_{2}^{\prime}}\left(S_{x_{1}}\right),
$$

which is thus measurable as a point-wise limit. Let $\mathfrak{M}$ be the set of $Q \in \mathbb{B}_{1} \times \mathbb{B}_{2}$ such that $Q \cap\left(A_{n}^{1} \times A_{n}^{2}\right) \in \Omega$ for all $n$, where $\left\{A_{n}^{i}\right\}$ are increasing sequences in $\mathbb{B}_{i}$ with $\bigcup_{i} A_{n}^{i}=X_{i}$ and $\lambda\left(A_{n}^{i}\right)$ bounded for all $n, i=1,2$. One has that

ג) $\mathfrak{M}$ contains all measurable rectangles, by (a).

$\beta) \mathfrak{M}$ is closed for finite disjoint unions, by (b).

$\gamma) \mathfrak{M}$ is a monotone class, by (c) and (e).

Since every intersection of measurable rectangles is a union of such, it follows from $\alpha$ ), $\beta$ ), $\gamma$ ) that $\mathfrak{M}=\mathbb{B}_{1} \times \mathbb{B}_{2}$. But $\mathfrak{M} \subset \Omega$ : thus $\Omega=\mathbb{B}_{1} \times \mathbb{B}_{2}$.

We need also the following obvious

Lemma A2. Let $Y, Y^{\prime}, Y_{1}, Y_{1}^{\prime}$ be measurable spaces with $X \in Y$ measurable and $\varphi: Y \rightarrow Y_{1}, \psi: Y_{1}^{\prime} \rightarrow Y^{\prime}$ measurable. Given a kernel $\lambda: Y \rightarrow Y^{\prime}$, we get kernels $\left.\lambda\right|_{X}: Y \rightarrow Y^{\prime}, \varphi^{*} \lambda: Y_{1} \rightarrow Y^{\prime}$ and $\psi_{*} \lambda: Y_{1}^{\prime} \rightarrow Y^{\prime}$ by setting

$$
\begin{gathered}
\left(\left.\lambda\right|_{X}\right)(f)=\lambda\left(\mathbb{1}_{X} \cdot f\right), f \in \overline{\mathscr{F}}+(X), \\
\left(\varphi^{*} \lambda\right)\left(g_{1}\right)=\lambda\left(g_{1} \circ \varphi\right), g_{1} \in \overline{\mathscr{F}}+\left(Y_{1}\right), \\
\left(\psi_{*} \lambda\right)(g)=\lambda(g) \circ \psi, g \in \overline{\mathscr{F}}+(Y) .
\end{gathered}
$$

Now the result we seek follows from the formula

$$
F_{\lambda}=\varphi^{*}\left\{\left.\left(\pi_{*} \tau^{*} \lambda \otimes \varepsilon\right)\right|_{S}\right\},
$$

where $\tau: \gamma \in \Gamma \rightarrow \gamma^{-1} \in \Gamma, \varepsilon$ is the identity kernel: $Z \rightarrow Z, S=\{(\gamma, z) \in \Gamma \times Z$; $s(\gamma)=\pi(z)\}$ and $\varphi:(\gamma, z) \in S \rightarrow \gamma z \in Z$.

\section{Appendix B. Examples}

\section{Group}

A (measurable) groupoid is a group iff $\Gamma^{(0)}$ has a single element $e$. The $\Gamma$-kernels are in this case the positive measures on $\Gamma$, with the convolution of $\Gamma$-kernels the usual 
convolution of measures on a group. The transverse functions are the left-invariant measures, i.e., the multiples of the left Haar measure $v$ in the locally compact group case. The convolution $*$ is then the usual convolution of functions on a group, and there is up to a constant a unique transverse $\mu$ - $\delta$-measure $\Lambda$, with $\Lambda_{v} \circ v=v$ and $\delta=\Delta$, the modular function on the group.

\section{Space}

If $\Gamma=\Gamma^{(0)}=X$, i.e. if all elements of $\Gamma$ are idempotent the $\Gamma$-kernels $\lambda$ coincide with the transverse functions and are, as follows, one-to-one with the measurable functions $f$ on $X$ : one has $\lambda^{x}=f \varepsilon_{x}, \varepsilon_{x}$ the Dirac measure at $x \in X$. Convolution in this case coincides with pointwise multiplication. The transverse measures are the positive measures on $X$. Physically this case corresponds to the classical limit of quantum mechanics.

\section{Space $\times$ Space}

If the measurable groupoid $\Gamma$ is both transitive (i.e., there is a $\gamma: x \rightarrow y$ to each pair $x$, $y \in \Gamma^{(0)}$ and unicursal (i.e., $\gamma, \gamma^{\prime}: x \rightarrow y$ implies that $\gamma^{\prime}=\gamma$ ), then $\Gamma=X \times X$ (as a measure space), where $X=\Gamma^{(0)}$. One has $r(y, x)=y, s(y, x)=x,(y, x)^{-1}=(x, y)$ and $(y, x)$ $(x, t)=(y, t), x, y, t \in X$. The $\Gamma$-kernels $\lambda$ are as follows: one-to-one with the kernels $\underline{\lambda}: X \rightarrow X$, one has $\lambda^{y}=\varepsilon_{y} \otimes \underline{\lambda}_{y}, y \in X$. The convolution of $\Gamma$-kernels $\lambda$ is obtained from the product of the corresponding kernels $\underline{\lambda}: \underline{\lambda * \mu}=\underline{\lambda} \circ \underline{\mu}$. $\lambda$ is $\sigma$-finite iff $\underline{\lambda}$ is $\sigma$-finite. For a transverse measure $v$ one has $\underline{v}_{y}=\underline{v}$ (independent of $y$ ), $\mathscr{E}^{+}$thus consists of the positive measures $\underline{v}$ on $X$, with $v$ faithful iff $\underline{y} \neq 0$. Convolution of functions is given by

$$
(f * g)(y, x)=\int f(y, t) g(t, x) d \underline{v}(t) .
$$

The modular functions $\delta$ of the form $\delta(y, x)=e^{V(y)} e^{-V(x)}, V$ a measurable function on $X$ : one has thus $v\left(\delta^{-1}\right)=\underline{v}\left(e^{V}\right) e^{-V}$. There is up to a constant a unique transverse measure: indeed with $v_{a}$ defined by $\underline{v}_{a}=\varepsilon_{a}, a \in X$, one has $v_{a} * v=v$ for all $v \varepsilon \mathscr{E}^{+}$, hence by (70)

$$
\Lambda^{\prime}(v)=\Lambda^{\prime}\left(v_{a} * v\right)=\Lambda_{v_{a}}\left(v\left(\delta^{-1}\right)\right)=k \underline{v}\left(e^{V}\right),
$$

where $k=\Lambda_{v_{a}}\left(e^{-v}\right)$. Since, for $\varphi \in \mathscr{F}^{+}(X), \underline{(\varphi \circ s) v}=\varphi \underline{v}$ one has $\Lambda_{v} \circ v=k\left(e^{v} \underline{v}\right) \otimes \underline{v}$.

\section{Groupoid of a Group Action}

With $(g \times x) \in G \times X \rightarrow g x \in X$ a measurable action of a measurable group $G$ on a locally compact space $X, \Gamma=X \times G$ is given the structure of a measurable groupoid as follows: one sets $\Gamma^{(0)}=X, r(y, g)=y, s(y, g)=g^{-1} y,(y, g)^{-1}=\left(g^{-1} y, g^{-1}\right)$, $(y, g)\left(g^{-1} y, h\right)=(y, g h), y \in X, g, h \in G$. The $\Gamma$-kernels $\lambda$ are one-to-one with kernels $\bar{\lambda}: G \rightarrow X:$ one has $\lambda^{y}=\varepsilon_{y} \otimes \bar{\lambda}_{y}, \sigma$-finiteness corresponding to $\sigma$-finiteness. The transverse functions thus correspond to the $\sigma$-finite kernels $\bar{v}_{s x}=s \bar{v}_{x}, x \in X, s \in G$. The convolution of functions on $\Gamma$ is given by

$$
(f \underset{v}{*} g)(y, s)=\int f(y, t) g\left(t^{-1} y, t^{-1} s\right) d \bar{v}_{y}(t) .
$$


If $G$ is locally compact with left Haar measure $\sigma$, one obtains a $v_{0} \in \mathscr{E}^{+}$by setting $(\vec{v})_{y}=\sigma, y \in X$. In that case and that of a continuous action of $G$ on a locally compact $X$, (A3) is the multiplication of the twisted product (convolution algebra) $C(X) \underset{\alpha}{X} G$ where $\left(\alpha_{t} f\right)(y)=f\left(t^{-1} y\right), f \in C(X)$ and one sets $f_{t}(y)=f(y, t)$, $y \in X, t \in G$. The modular functions $\delta$ on $\Gamma$ are characterized by the "cocycle condition"

$$
\delta(y, s t)=\delta(y, s) \delta\left(s^{-1} y, t\right), y \in X, s, t \in G .
$$

The transverse $\Gamma-\delta$ measures are then characterized as follows for a locally compact group $G$ : a positive measure $M$ on $X$ is of the form $M=\Lambda_{\sigma}$ iff it fulfills

$$
d M\left(s^{-1} x\right)=\Delta(s) \delta(x, s)^{-1} d M(x), x \in X, s \in G,
$$

where $\Delta$ is the modular function of $G$ : if $m=\Lambda_{v_{0}} \circ v_{0}$ one has indeed, for $f \in \mathscr{F}^{+}(X \times G)$

$$
\begin{aligned}
m(f) & =\int f(x, g) d M(x) d \sigma(g)=m\left(\delta^{-1} \tilde{f}\right) \\
& =\int \delta^{-1}(g x, g) f\left(x, g^{-1}\right) d M(g x) d \sigma(g) \\
& =\int \delta(x, g) \Delta(g)^{-1} f(x, g) d M\left(g^{-1} x\right) d \sigma(g) .
\end{aligned}
$$

\section{Graph of an Equivalence Relation}

This case is that of a unicursal measurable groupoid $\Gamma$ (if $X=\Gamma^{(0)}, \Gamma \subset X \times X$ is then the graph of the equivalence relation). We assume that $X$ is a standard measurable space within which $\Gamma$, as well as the diagonal in $X \times X$, are measurable; and denote $\Omega$ the set of equivalence classes in $X$. One obtains a measurable groupoid structure on $\Gamma$ by considering $\Gamma$ as a subgroupoid of the groupoid $X \times X$ as defined in Example 3 above (we denote the latter $\Gamma^{\prime}$ ). With $i$ the injection: $\Gamma \subset \Gamma^{\prime}, \pi$ the canonical map: $X \rightarrow \Omega, f \in \overline{\mathscr{F}}^{+}(\Gamma)$ and $\lambda$ a kernel: $\Gamma \rightarrow X$ the definitions

$$
\left.\begin{array}{l}
f^{\prime}(x, y)=\mathbb{1}_{\Gamma}(x, y) f(x, y), \quad x, y \in X, \\
\lambda^{\prime}(g)=\lambda(g \circ i), \quad g \in \overline{\mathscr{F}}+(\Gamma),
\end{array}\right\}
$$

yield bijections $f \rightarrow f^{\prime}, \lambda \rightarrow \lambda^{\prime}$ onto the measurable functions on $\Gamma^{\prime}$ vanishing outside $\Gamma$, respectively the kernels: $\Gamma^{\prime} \rightarrow X$ carried by $\Gamma^{\prime}$ (whereby ( $\sigma$-finite) $\Gamma$-kernels are turned into $\sigma$-finite $\Gamma^{\prime}$-kernels). Using these bijections the operations $f \rightarrow \tilde{f}$, $(\lambda, f) \rightarrow \lambda * f,\left(\lambda_{1}, \lambda_{2}\right) \rightarrow \lambda_{1} * \lambda_{2}$ can then be performed either within $\Gamma$ or within $\Gamma^{\prime}$ for the corresponding primed objects. Given a ( $\sigma$-finite) $\Gamma$-kernel $\lambda$, we then define the kernel $\lambda: X \rightarrow X$ by

$$
\underline{\lambda}(\varphi)=\lambda(\varphi \circ s), \quad \varphi \in \overline{\mathscr{F}}^{+}(X),
$$

thus getting a bijection $\lambda \rightarrow \underline{\lambda}$ onto the kernels $\underline{\lambda}: X \rightarrow X$ such that $\underline{\lambda}_{y}, y \in X$, is carried by the equivalence class of $y$ (the inverse map is given by $\left.\lambda^{y}=\left.\left(\varepsilon_{y} \otimes \underline{\lambda}_{y}\right)\right|_{\Gamma}\right)$. Then $v=\left.(\varepsilon \otimes \underline{v})\right|_{\Gamma}$ is a transverse function on $\Gamma$ iff $\underline{v}_{y}=\underline{v}_{x}$ whenever $\pi(y)=\pi(x)$. These transverse functions are one-to-one with the kernels $\underset{\sim}{y}: X \rightarrow \Omega$ fibered by $\pi$ and $\operatorname{id}_{\Omega}$, with the bijection $v_{\lambda}=y_{\pi(x)}, x \in X$.

The easiest (so-called smooth) case of equivalence relations is that where the quotient measurable structure on $\Omega$ is countably separated (for that matter, 
standard), also characterized by the existence of kernels $\sigma: \Omega \rightarrow X$ with $\sigma_{q}$ bounded nonvanishing for all $q \in \Omega$. Let then $\delta=\left.\left(e^{V} \otimes e^{-V}\right)\right|_{\Gamma}, V \in \mathscr{F}^{+}(X)$ (so that $\lambda(\delta)=e^{V} \lambda\left(e^{-V}\right)$ for the $\Gamma$-kernels $\lambda$ ). There is then a bijection $\Lambda \rightarrow \varrho_{A}$ from the $\Gamma$ - $\delta$-functions onto the bounded measures on $\Omega$ given by $\Lambda(v)=\left\langle\varrho_{A}, v\left(e^{V}\right)\right\rangle, \quad v \in \mathscr{E}^{+}$. One has $\varrho_{\Lambda}=\pi\left(e^{-V} \Lambda_{v_{0}}\right)$ for any $v_{0} \in \mathscr{E}^{+}$ with $v_{0}\left(\mathbb{1}_{\Gamma}\right)=\mathbb{1}_{X}$. For $v \in \mathscr{E}^{+}$one has $\Lambda_{v}=\varrho_{\nu} \circ\left(e^{V} v\right)$, in other terms

$$
\Lambda_{v}=\int_{\Omega} d \varrho_{\Lambda}(q) e^{-V} \underline{\sim}_{q}
$$

Acknowledgements. I am indebted to A. Connes for communication of an early draft of his manuscript and friendly informative discussions; to H. Araki for mentioning Lemma 2, to R. Arens and C. Amaral for remarks.

This paper was written whilst a guest of the Mathematics Department of UCLA. I am indebted to Professors T. Gamelin, A. Hales, and M. Takesaki for their warm hospitality; and to Ms. Phyllis Parris for her enduring help in producing the manuscript. I thank Prof. Kuiper for the hospitality of I.H.E.S. in the fall of 1979.

\section{References}

1. Connes, A.: Sur la theorie noncommutative de l'integration. In: Lecture Notes in Mathematics, Vol. 725. Berlin, Heidelberg, New York: Springer 1978

2. Takesaki, M.: Tomita's theory of modular Hilbert algebras and its applications. In: Lecture Notes in Mathematics, Vol. 128. Berlin, Heidelberg, New York: Springer 1970

Communicated by R. Jost

Received December 11, 1981 